\title{
Normal Contact with Adhesion
}

\subsection{Introduction}

Between any two electrically neutral bodies there exist relatively weak interaction forces which rapidly decline with increasing distance between the bodies. These forces are known as adhesive forces and, in most cases, cause a mutual attraction. Adhesive forces play an essential role in many technical applications. It is the adhesive forces that are responsible for the behavior of glue, for instance. Adhesive tape, self-adhesive envelopes, etc., are further examples of adhesive forces. They are of particular importance for applications where one of the following conditions is met:

- The surfaces of the bodies are very smooth (e.g., the magnetic disc of a hard drive).

- One of the contact partners is made of a soft material (rubber or biological structures).

- We are dealing with a microscopic system, in which adhesive forces generally have a larger influence than body forces, because the body and surface forces are scaled differently (micro-mechanical devices, atomic force microscope, biological structures, etc.).

At a microscopic scale, the adhesive forces are determined by the type of the interaction potential. It is possible to define a characteristic "range" of adhesive forces based on the specific type of the potential. However, as Griffith already demonstrated in his famous work on the theory of cracks (Griffith 1921), the most important parameter is not solely the magnitude of the interactions or their range, but instead the product of both; i.e., the work required separation of the surfaces. This work per unit surface area is referred to as the work of adhesion or effective relative surface energy, $\Delta \gamma$, of the contacting bodies. 
Griffith's theory is based on the energy balance between the elastic energy released due to an advancement of the crack boundary and the required work of adhesion. It is assumed that there are no interaction forces beyond the contact area, which corresponds to the assumption of a vanishingly low range of the adhesive forces. This assumption is valid for real adhesive interactions if the range of the adhesive forces is much smaller than any characteristic length of the contact. The theory of adhesive contact published in 1971 by Johnson, Kendall, and Roberts is valid under the same conditions as Griffith's theory: for the vanishingly low range of the interactions. In this chapter, when we refer to the theory of adhesive contacts in the "JKR approximation", it is understood to mean the vanishingly low range of the adhesive forces. In JKR theory, the work of adhesion is also the sole parameter of the adhesive interaction. Among other results, the adhesive force between a sphere of radius $R$ and an elastic half-space is given by the equation:

$$
F_{A}=\frac{3}{2} \Delta \gamma \pi R
$$

In micro-systems, situations can arise where the range of the adhesive forces is of the same length as the smallest characteristic length of the contact (usually the indentation depth), or even greater than the characteristic contact length. The simplest of such cases is the contact of hard spheres with weak interaction forces, where the elastic deformation is negligible. This case was examined and published by Bradley (1932). Bradley calculated the adhesive force between a rigid plane and a rigid sphere of radius $R$. He assumed the existence of van der Waals forces acting between the molecules of both bodies, decreasing proportionally to $1 / r^{7}$ with increasing distance between the molecules (which corresponds to the attractive component of the Lennard-Jones potential). For the contact of a rigid plane and a rigid sphere, the adhesive force equals:

$$
F_{A}=2 \Delta \gamma \pi R
$$

This equation also only features the separation energy and does not account for the coordinate dependency of the interaction. It can be easily shown that the result from Bradley's approximation is invariant with regards to the exact coordinate dependency of the interaction potential, as long as the half-space approximation is valid.

The logical extension of Bradley's model lies in the consideration of the elastic deformations caused by long-range adhesive interactions. An approximate solution for this problem was only discovered about 40 years after Bradley's publication by Derjaguin, Muller, and Toporov (1975) (DMT theory). While the approximation does take into account the adhesive forces, the elastic deformation of the surfaces is, nonetheless, calculated with the solution by Hertz (1882) for non-adhesive contacts. Under these assumptions, Derjaguin, Muller, and Toporov arrived at the same equation for the adhesive force as Bradley, stating: 
The van der Waals' forces are capable of increasing the area of elastic contact of the ball with the plane, yet it has been shown that the force, as required for overcoming the van der Waals' forces and breaking up the contact, does not increase thereby and may be calculated, if one considers the point contact of a non-deformed ball with a plane.

To judge whether the adhesive interactions are short or long-ranged (thus determining whether the adhesive contact is of the "JKR type" or "DMT type"), the characteristic displacement of the bodies until the separation of the adhesive contact (the characteristic value for the height of the adhesive "neck") $\left(R(\Delta \gamma)^{2} /\left(E^{*}\right)^{2}\right)^{1 / 3}$ (see (3.45)) should be compared to the characteristic range of the adhesive interactions, $z_{0}$. This leads to the parameter:

$$
\mu:=\frac{R(\Delta \gamma)^{2}}{\left(E^{*}\right)^{2} z_{0}^{3}},
$$

which was initially introduced by Tabor (1977) and is known as the "Tabor parameter". For neck heights much greater than the range of the interactions (large Tabor parameter), the range can be considered vanishingly low. This limiting case leads to the JKR theory. The other limiting case is the DMT theory.

Maugis's (1992) theory of a contact with Dugdale's (1960) simple model for the interaction potential was of great methodological interest for the theory of adhesive contacts. Maugis assumed that the adhesive stress between surfaces remains constant up to a certain distance $h$ and then drops abruptly. For this case the specific work of adhesion equals:

$$
\Delta \gamma=\sigma_{0} h
$$

While the coordinate dependency of the Dugdale potential is not realistic, this bears little importance for most adhesive problems since both limiting cases-JKR and DMT - are independent of the exact type of the interaction potential. Under these conditions, even the simplest model of interaction is valid and informative. The great advantage of the Dugdale potential lies in the fact that it allows a mostly analytical solution of the problem. Maugis's theory not only provided a representation of the two limiting cases but also an explanation of the transition between the JKR and DMT theories.

Since the exact form of the interaction potentials is insignificant for the adhesion (as long as the work of adhesion is defined and remains constant), Greenwood and Johnson (1998) developed a theory which represented the stress distribution in the adhesive contact as the superposition of two Hertzian stress distributions of different radii. Compared to Maugis's theory, this represented a vast "trivialization" of the involved contact mechanics. It should be noted that the "double Hertz" solution corresponds to a rather strange interaction potential. But since the exact form of the interaction potential is insignificant, the theory of Greenwood and Johnson represents an interesting alternative to Maugis's theory. It too features the JKR and DMT models as its limiting cases. 
The two most well-known theories of adhesion by JKR and DMT both lead to an adhesive force which is independent of the elastic properties of the contacting media. To avoid any misunderstanding, it should be expressly noted that this simple property only applies to parabolic contacts. In no way can this lack of dependency on the elastic moduli be generalized to arbitrary adhesive contacts.

The consideration of the tangential stresses in the adhesive contact requires another general remark. Both JKR and DMT theory are based on the Hertzian theory of frictionless contact. It is surely a valid and self-consistent model assumption. From the physical point of view, on the contrary, this assumption is rather questionable. Physically, the JKR limiting case implies an infinitely strong yet infinitely short-ranged interaction. This means that the surfaces of the adhesive JKR contact are pressed together under infinitely low ranged but infinitely strong stress, which undermines the notion of a "frictionless" contact. However, for practical applications, the difference between frictional or frictionless adhesive contact is relatively limited and can be considered negligible in most cases.

The following two sections will present two alternative approaches to the solution of the adhesive normal contact problem. The first approach is the reduction of the adhesive normal contact problem to the non-adhesive one, and the second approach is the direct solution utilizing the MDR.

\subsection{Solution of the Adhesive Normal Contact Problem by Reducing to the Non-Adhesive Normal Contact Problem}

The basic idea of the theory of adhesive contact by Johnson, Kendall, and Roberts (1971) is the same as the one of Griffith's theory of cracks. In their frequently cited paper they write:

... the approach followed in this analysis, is similar to that used by Griffith in his criterion for the propagation of a brittle crack.

The idea is based on the consideration of energy balance between the elastic energy and the surface energy during the propagation of the crack or the boundary of the adhesive contact. Since the surface energy of an axisymmetric contact is trivially determined from the contact area, the only remaining non-trivial problem lies in calculating the elastic energy of the adhesive contact. This can always be done if the solution of the respective non-adhesive normal contact problem is known. The JKR method to calculate the energy is the second important point of the classic paper. It is also ingeniously simple and based on the assertion that the adhesive contact can be represented as a superposition of a non-adhesive contact and a rigid body translation. Perhaps the easiest way to imagine this is to consider the contact between a rigid indenter with the profile $\tilde{z}=f(r)$ and an elastic half-space. We obtain the configuration of the adhesive contact by initially indenting the elastic half-space (without regard for adhesion), causing it to form a contact area of radius $a$, and raising the entire contact area after that, without change in contact radius. 
Furthermore, we will assume the solution of the non-adhesive contact problem, particularly the relationships between the indentation depth, the contact radius, and the normal force. Any quantity of the triple $\left\{F_{N}, a, d\right\}$ uniquely defines the others. It will prove advantageous to describe the normal force and the indentation depth as functions of the contact radius:

$$
F_{N}=F_{N, \text { n.a. }}(a), \quad d=d_{\text {n.a. }}(a)
$$

The indices "n.a." indicate that these are the solutions of the non-adhesive contact problem. These equations also imply that the dependency of the force on the indentation depth is known. We obtain the incremental contact stiffness $k_{\text {n.a. }}$ by differentiating the force with respect to $d$ and the elastic energy $U_{\text {n.a. }}$ by integrating with respect to $d$. These quantities can also be rewritten as functions of the contact radius:

$$
k_{\text {n.a. }}=k_{\text {n.a. }}(a), \quad U_{\text {n.a. }}=U_{\text {n.a. }}(a) .
$$

In the following all functions for (3.5) and (3.6) are considered to be known.

Let us now indent the profile to a contact radius $a$. The elastic energy of this state is $U_{\text {n.a. }}(a)$, the indentation depth $d_{\text {n.a. }}(a)$, and the force $F_{N \text {,n.a. }}(a)$. In the second step, we lift the profile by $\Delta l$ without changing the contact radius. The stiffness (only dependent on the radius) remains constant for this process and equals $k_{\text {n.a. }}(a)$. The force is then given by:

$$
F_{N}(a)=F_{N, \text { n.a. }}(a)-k_{\text {n.a. }}(a) \Delta l,
$$

and the potential energy is:

$$
U(a)=U_{\text {n.a. }}(a)-F_{N, \text { n.a. }}(a) \Delta l+k_{\text {n.a. }}(a) \frac{\Delta l^{2}}{2} .
$$

The new indentation depth at the end of the process equals:

$$
d=d_{\text {n.a. }}(a)-\Delta l
$$

Obtaining $\Delta l$ from (3.9) and by substituting it into (3.8), we obtain the potential energy:

$$
U(a)=U_{\text {n.a. }}(a)-F_{N, \text { n.a. }}(a)\left(d_{\text {n.a. }}(a)-d\right)+k_{\text {n.a. }}(a) \frac{\left(d_{\text {n.a. }}(a)-d\right)^{2}}{2} .
$$

The total energy (under consideration of the surface energy) now equals:

$$
\begin{aligned}
U_{\text {tot }}(a)= & U_{\text {n.a. }}(a)-F_{N, \text { n.a. }}(a)\left(d_{\text {n.a. }}(a)-d\right) \\
& +k_{\text {n.a. }}(a) \frac{\left(d_{\text {n.a. }}(a)-d\right)^{2}}{2}-\pi a^{2} \Delta \gamma
\end{aligned}
$$


The equilibrium value of the contact radius follows from the total energy minimum condition (for constant indentation depth $d$ ):

$$
\begin{aligned}
\frac{\partial U_{\text {tot }}(a)}{\partial a}= & \frac{\partial U_{\text {n.a. }}(a)}{\partial a}-\frac{\partial F_{N, \text { n.a. }}(a)}{\partial a} \Delta l-F_{N, \text { n.a. }}(a) \frac{\partial d_{\text {n.a. }}(a)}{\partial a} \\
& +\frac{\partial k_{\text {n.a. }}(a)}{\partial a} \frac{\Delta l^{2}}{2}+k_{\text {n.a. }}(a) \Delta l \frac{\partial d_{\text {n.a. }}(a)}{\partial a}-2 \pi a \Delta \gamma \\
= & \left(\frac{\partial U_{\text {n.a. }}(a)}{\partial a}-F_{N, \text { n.a. }}(a) \frac{\partial d_{\text {n.a. }}(a)}{\partial a}\right) \\
& -\left(\frac{\partial F_{N, \text { n.a. }}(a)}{\partial a} \Delta l-k_{\text {n.a. }}(a) \Delta l \frac{\partial d_{\text {n.a. }}(a)}{\partial a}\right) \\
& +\frac{\partial k_{\text {n.a. }}(a)}{\partial a} \frac{\Delta l^{2}}{2}-2 \pi a \Delta \gamma \\
=0 . &
\end{aligned}
$$

The terms in parentheses disappear and the equation takes the form:

$$
\frac{\partial k_{\mathrm{n} . \mathrm{a}}(a)}{\partial a} \frac{\Delta l^{2}}{2}=2 \pi a \Delta \gamma \text {. }
$$

It follows that:

$$
\Delta l=\sqrt{\frac{4 \pi a \Delta \gamma}{\frac{\partial k_{\text {n.a. }}(a)}{\partial a}}} \quad \text { (for the general axially symmetric case) }
$$

Substituting this quantity into (3.9) and (3.7) yields an equation for determining the relationship between the indentation depth, the contact radius, and the normal force:

$$
\begin{gathered}
d=d_{\text {n.a. }}(a)-\sqrt{\frac{4 \pi a \Delta \gamma}{\frac{\partial k_{\text {n.a. }}(a)}{\partial a}}} \quad \text { (for an arbitrary medium), } \\
F_{N}(a)=F_{N, \text { n.a. }}(a)-k_{\text {n.a. }}(a) \sqrt{\frac{4 \pi a \Delta \gamma}{\frac{\partial k_{\text {n.a. }}(a)}{\partial a}}} \quad \text { (for an arbitrary medium). }
\end{gathered}
$$

It becomes apparent that the three functions which directly (and in its entirety) determine the solution of the adhesive contact problem are the three dependencies of the non-adhesive contact: indentation depth as a function of the contact radius, normal force as a function of the contact radius, and therefore the incremental stiffness as a function of the contact radius. The latter quantity equals the stiffness for the indentation by a circular cylindrical indenter of radius $a$. It should be noted that these are general equations and not subject to the homogeneity of the medium (neither in-depth nor in the radial direction). As such, they also apply to layered or functionally graded media. The sole condition for the validity of (3.15) and (3.16) is the conservation of rotational symmetry during indentation. 
For a homogeneous medium, the equations can be simplified even further. Here, the stiffness is given by $k_{\text {n.a. }}=2 E^{*} a$, and we obtain:

$$
\Delta l(a)=\sqrt{\frac{2 \pi a \Delta \gamma}{E^{*}}} \quad \text { (for a homogeneous medium). }
$$

The determining equations (3.15) and (3.16) take on the form:

$$
\begin{gathered}
d=d_{\text {n.a. }}(a)-\sqrt{\frac{2 \pi a \Delta \gamma}{E^{*}}} \quad(\text { for a homogeneous medium), } \\
F_{N}(a)=F_{N \text {,n.a. }}(a)-\sqrt{8 \pi E^{*} \Delta \gamma a^{3}} \quad \text { (for a homogeneous medium). (3.18) }
\end{gathered}
$$

Naturally, the pressure distribution in an adhesive contact and the displacement field outside the contact area are also composed of the two solutions of the non-adhesive contact problem: the solutions for the non-adhesive indentation by $d_{\text {n.a. }}(a)$ and the subsequent rigid retraction by $\Delta l$. Let us denote the stress distribution and the displacement field for the non-adhesive contact problem by $\sigma(r ; a)_{\text {n.a. }}$ and $w(r ; a)_{\text {n.a., }}$, respectively. With the stress and the displacement field for a rigid translation given by (2.22), the stress distribution and the displacement for the adhesive contact problem are then represented by the following equations:

$$
\begin{aligned}
& \sigma(r ; a)=\sigma(r ; a)_{\text {n.a. }}+\frac{E^{*} \Delta l}{\pi \sqrt{a^{2}-r^{2}}}, \quad r<a, \\
& w(r ; a)=w(r ; a)_{\text {n.a. }}-\frac{2 \Delta l}{\pi} \arcsin \left(\frac{a}{r}\right), \quad r>a,
\end{aligned}
$$

or after inserting (3.17):

$$
\begin{array}{r}
\sigma(r ; a)=\sigma(r ; a)_{\text {n.a. }}+\sqrt{\frac{2 E^{*} a \Delta \gamma}{\pi}} \frac{1}{\sqrt{a^{2}-r^{2}}}, \\
r<a(\text { for a homogeneous medium }), \\
w(r ; a)=w(r ; a)_{\text {n.a. }}-\sqrt{\frac{8 \pi a \Delta \gamma}{\pi E^{*}}} \arcsin \left(\frac{a}{r}\right), \\
r>a \text { (for a homogeneous medium). }
\end{array}
$$

Equations (3.18) and (3.20) completely solve the adhesive normal contact problem. The magnitude of the force of adhesion is of particular interest. We will define it as the maximum pull-off force required to separate bodies. In mathematical 
terms, this means the maximum pull-off force for which there still exists a stable equilibrium solution of the normal contact problem.

Another important quantity to consider is the force in the last possible state of stable equilibrium, after which the contact dissolves entirely. This force depends on the character of the loading conditions. We commonly distinguish between the limiting cases of force-controlled and displacement-controlled trials. The stability condition for force-control is given by:

$$
\left.\frac{\mathrm{d} F_{N}}{\mathrm{~d} a}\right|_{a=a_{c}}=0
$$

and for displacement-control case by:

$$
\left.\frac{\mathrm{d} d}{\mathrm{~d} a}\right|_{a=a_{c}}=0 .
$$

The conditions (3.21) and (3.22) can be consolidated into the condition

$$
\left.\frac{\mathrm{d} d_{\text {n.a. }}(a)}{\mathrm{d} a}\right|_{a=a_{c}}=\xi \sqrt{\frac{\pi \Delta \gamma}{2 E^{*} a_{c}}}, \quad \xi= \begin{cases}3, & \text { force-control, } \\ 1, & \text { displacement-control, }\end{cases}
$$

from which we can determine the critical contact radius, where the contact detaches (see Sect. 3.3 for a full derivation). The respective critical values of the indentation depth and normal force are obtained by substituting this critical radius into (3.18).

\subsection{Direct Solution of the Adhesive Normal Contact Problem in the Framework of the MDR}

An alternative to the reduction to the non-adhesive contact problem described in the previous section is provided by the MDR (see Popov and Heß 2015, for example), which presents an immediate solution to the adhesive normal contact problem. This alternative approach can, for example, be of interest for complex dynamic loading conditions requiring a numerical implementation.

The calculation method via the MDR consists of the following steps:

- In the first step the given three-dimensional profile $\tilde{z}=f(r)$ is transformed to an equivalent plane profile $g(x)$ via (2.6):

$$
g(x)=|x| \int_{0}^{|x|} \frac{f^{\prime}(r)}{\sqrt{x^{2}-r^{2}}} \mathrm{~d} r .
$$



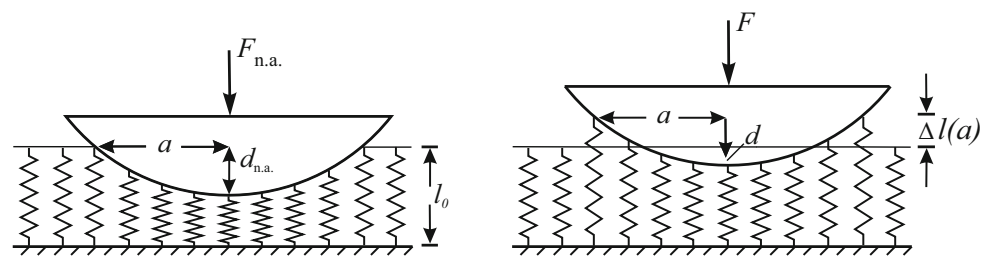

Fig.3.1 Qualitative representation of the indentation and lifting process of a spherical 1D-indenter with an elastic foundation, which exactly models the properties of the adhesive contact between a rigid spherical indenter and an elastic half-space

- The profile $g(x)$ is now pushed into the one-dimensional elastic foundation defined, according to (2.5):

$$
\Delta k_{z}=E^{*} \Delta x
$$

until a contact radius $a$ is reached. Until this point, the adhesion will not be considered. This process is depicted in Fig. 3.1.

- In the third step, the indenter is lifted up. It is assumed that all springs involved in the contact adhere to the indenter-the contact radius thus remains constant. In this process, the springs at the edge experience the maximum increase in tension. Upon reaching the maximum possible elongation (3.17) of the outer springs

$$
\Delta l(a)=\sqrt{\frac{2 \pi a \Delta \gamma}{E^{*}}}
$$

they detach. This criterion (3.17) was discovered by Heß (2010), and is known as the rule of Heß. A derivation of this criterion can be found in the appendix (see (11.31)).

The corresponding equilibrium described by the three quantities $\left\{F_{N}, d, a\right\}$ coincides exactly with the one of the three-dimensional adhesive contact.

The only difference to the algorithm for the non-adhesive contact (described in Chap. 2) lies in the modification of the indentation depth formula. The displacement of the outer springs is no longer zero but instead negative, with the absolute value equal to the critical value: $w_{1 D}(a)=-\Delta l(a)$. It follows that:

$$
d=g(a)-\Delta l(a)
$$

The normal force is once again given by the equation:

$$
F_{N}=2 E^{*} \int_{0}^{a}[d-g(x)] \mathrm{d} x .
$$

The only difference to the non-adhesive problem lies in the differing contact radius. 
Equation (3.27) determines the equilibrium configuration. The stability of this equilibrium is governed by the sign of the derivative $\mathrm{d}(\Delta l(a)+d-g(a)) / \mathrm{d} a$ :

$$
\begin{array}{ll}
\frac{\mathrm{d}}{\mathrm{d} a}(\Delta l(a)+d-g(a))>0, & \text { stable equilibrium, } \\
\frac{\mathrm{d}}{\mathrm{d} a}(\Delta l(a)+d-g(a))<0, & \text { unstable equilibrium, } \\
\frac{\mathrm{d}}{\mathrm{d} a}(\Delta l(a)+d-g(a))=0, & \text { critical state. }
\end{array}
$$

In general, the stability depends on the type of boundary condition employed for the indenter. The two limiting cases are force-controlled trials (which correspond to an infinitely soft test system) and displacement-controlled trials (which correspond to a rigid test system).

\section{Stability Condition for Displacement-Controlled Trials}

In this case, the indentation depth is constant and (3.29) for the critical state is:

$$
\left.\frac{\mathrm{d} g(a)}{\mathrm{d} a}\right|_{a=a_{c}}=\left.\frac{\mathrm{d} \Delta l(a)}{\mathrm{d} a}\right|_{a=a_{c}}=\sqrt{\frac{\pi \Delta \gamma}{2 E^{*} a_{c}}} .
$$

\section{Stability Condition for Force-Controlled Trials}

When the force is kept constant we must consider the varying indentation depth. The relationship between the indentation depth and the normal force is given by (3.28). Differentiating (3.28) under the condition $F_{N}=$ const yields:

$$
\begin{aligned}
\mathrm{d} \int_{0}^{a}[d-g(x)] \mathrm{d} x & =\mathrm{d} a \cdot \frac{\partial}{\partial a} \int_{0}^{a}[d-g(x)] \mathrm{d} x+\mathrm{d} d \cdot \frac{\mathrm{d}}{\mathrm{d} d} \int_{0}^{a}[d-g(x)] \mathrm{d} x \\
& =\mathrm{d} a \cdot[d(a)-g(a)]+\mathrm{d} d \cdot a \\
& =-\mathrm{d} a \cdot \Delta l(a)+\mathrm{d} d \cdot a=0 .
\end{aligned}
$$

It follows that:

$$
\left.\frac{\mathrm{d} d}{\mathrm{~d} a}\right|_{F_{N}=\mathrm{const}}=\frac{\Delta l(a)}{a} .
$$

The condition for the critical state (3.29) takes on the form of:

$$
\frac{\mathrm{d} \Delta l(a)}{\mathrm{d} a}+\frac{\mathrm{d} d}{\mathrm{~d} a}-\frac{\mathrm{d} g(a)}{\mathrm{d} a}=\frac{\mathrm{d} \Delta l(a)}{\mathrm{d} a}+\frac{\Delta l(a)}{a}-\frac{\mathrm{d} g(a)}{\mathrm{d} a}=0,
$$

or

$$
\left.\frac{\mathrm{d} g(a)}{\mathrm{d} a}\right|_{a=a_{c}}=\left[\frac{\Delta l(a)}{a}+\frac{\mathrm{d} \Delta l(a)}{\mathrm{d} a}\right]_{a=a_{c}}=3 \sqrt{\frac{\pi \Delta \gamma}{2 E^{*} a_{c}}} .
$$


The stability conditions can now be combined into a single equation:

$$
\left.\frac{\mathrm{d} g(a)}{\mathrm{d} a}\right|_{a=a_{c}}=\xi \sqrt{\frac{\pi \Delta \gamma}{2 E^{*} a_{c}}}, \quad \xi=\left\{\begin{array}{l}
3, \text { force-control, } \\
1, \text { displacement-control. }
\end{array}\right.
$$

The transformation rules of the MDR for the pressure distribution and the displacements—see (2.13) and (2.14) — remain valid for the adhesive contact.

\subsection{Areas of Application}

Adhesion can be desirable (such as in bonded connections, in adhesive medical bandages, as well as in many biological systems) or equally undesirable. The systems in the first group, where adhesion is desirable, are often inspired by biological systems and especially concern contacts with concave profiles (see Sect. 3.5.6). The study of the tiny hairs optimized for their adhesive functions on the limbs of (for example) geckos or insects has inspired technical solutions for achieving extreme adhesive effects.

Adhesion is also a major consideration in the design of micro-systems. One example is the contact between a measuring tip and the sample surface in atomic force microscopy. The indenting measuring tip often has a conical (see Sect. 3.5.2) or spherical (see Sects. 3.5.3 and 3.5.4) shape, or imperfect variations of these (see Sects. 3.5.5, 3.5.8, 3.5.9, 3.5.11, or 3.5.12). For assembly of nano- or microscopic systems the smallest flat indenters are utilized, which we will explore in their perfect (see Sect. 3.5.1) or imperfect form (see Sects. 3.5.10 and 3.5.13).

Any given sufficiently differentiable indenter profile can be represented as a Taylor series. Thus, we will provide the solution of the contact problem involving the power-law profile as a basic building block of the solution for such an arbitrary profile (see Sect. 3.5.7).

In addition, real surfaces are unavoidably rough. In Sect. 3.5.14 we will present a simple model of periodic roughness, which quite clearly illustrates the effect roughness has on the adhesive interaction.

\subsection{Explicit Solutions for Axially Symmetric Profiles in JKR Approximation}

\subsubsection{The Cylindrical Flat Punch}

The solution for the adhesive normal contact with a cylindrical flat punch was dicovered by Kendall (1971). In the JKR theory, adhesion is interpreted as an indentation by a flat cylindrical punch with a contact radius $a$, with the superposition of two indenter solutions of identical radius yielding a new indenter solution. Therefore, the solutions of the contact problem concerning the flat cylindrical punch are identical 
with and without adhesion. The results for the normal force $F_{N}$, the normal stresses $\sigma_{z z}$, and the displacements of the half-space surface $w$, are then given by:

$$
\begin{aligned}
F_{N}(d) & =2 E^{*} d a, \\
\sigma_{z z}(r ; d) & =-\frac{E^{*} d}{\pi \sqrt{a^{2}-r^{2}}}, \quad r \leq a, \\
w(r ; d) & =\frac{2 d}{\pi} \arcsin \left(\frac{a}{r}\right), \quad r>a .
\end{aligned}
$$

The only difference compared to the non-adhesive contact is that the adhesive case also permits negative indentation depths. The critical radius is predetermined by the indenter radius $a$. The critical indentation depth $d_{c}$ and critical normal force $F_{c}$, where stability of the contact is lost, are calculated from (3.18), independently of whether a force-controlled or displacement-controlled trial is being considered:

$$
\begin{aligned}
& d_{c}=-\sqrt{\frac{2 \pi a \Delta \gamma}{E^{*}}}, \\
& F_{c}=-\sqrt{8 \pi a^{3} E^{*} \Delta \gamma} .
\end{aligned}
$$

\subsubsection{The Cone}

The solution to the contact problem depicted in Fig. 3.2 was first found by Maugis and Barquins (1981). They used the general solution of the non-adhesive problem and the concept of energy release rate from linear fracture mechanics. The contact problem is completely solved by specifying the indentation depth $d$, the normal force $F_{N}$, the stress distribution $\sigma_{z z}$, and the displacements $w$; each as functions of the contact radius $a$. With the results from the previous Chapter (Sect. 2.5.2) and (3.18) and (3.20) we get:

$$
\begin{aligned}
d(a) & =\frac{\pi}{2} a \tan \theta-\sqrt{\frac{2 \pi a \Delta \gamma}{E^{*}}}, \\
F_{N}(a) & =\frac{\pi a^{2}}{2} E^{*} \tan \theta-\sqrt{8 \pi a^{3} E^{*} \Delta \gamma} \\
\sigma_{z z}(r ; a) & =-\frac{E^{*} \tan \theta}{2} \operatorname{arcosh}\left(\frac{a}{r}\right)+\sqrt{\frac{2 E^{*} \Delta \gamma}{\pi a}} \frac{a}{\sqrt{a^{2}-r^{2}}}, \quad r \leq a, \\
w(r ; a) & =\tan \theta\left(\sqrt{r^{2}-a^{2}}-r\right)+\left(a \tan \theta-\sqrt{\frac{8 a \Delta \gamma}{\pi E^{*}}}\right) \arcsin \left(\frac{a}{r}\right), \\
r>a . &
\end{aligned}
$$

Here, $\theta$ denotes the slope angle of the cone. The relationship between contact radius and indentation depth in the case without adhesion is given by:

$$
d_{\text {n.a. }}(a)=\frac{\pi}{2} a \tan \theta \text {. }
$$


Fig. 3.2 Adhesive normal contact between a rigid conical indenter and an elastic half-space

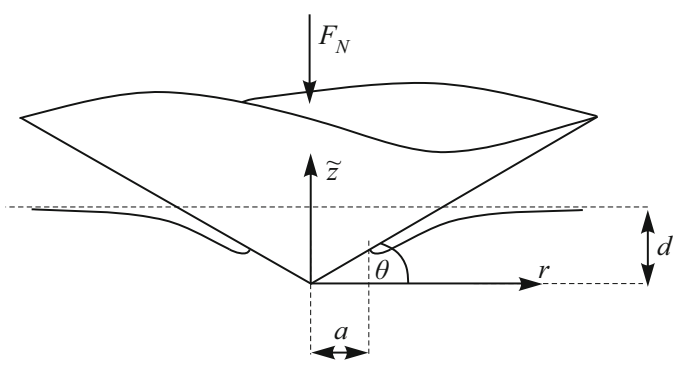

Therefore, for the critical contact radius, (3.23) results in:

$$
a_{c}=\frac{2 \xi^{2} \Delta \gamma}{\pi E^{*} \tan ^{2} \theta} .
$$

The critical values for the indentation depth and normal force are then:

$$
\begin{aligned}
d_{c} & =d_{\text {n.a. }}\left(a_{c}\right)-\sqrt{\frac{2 \pi a_{c} \Delta \gamma}{E^{*}}}=\frac{\left(\xi^{2}-2 \xi\right) \Delta \gamma}{E^{*} \tan \theta}, \\
F_{c} & =\frac{2 \Delta \gamma^{2}\left(\xi^{4}-4 \xi^{3}\right)}{\pi E^{*} \tan ^{3} \theta} .
\end{aligned}
$$

Here it must be specified whether the experiment is carried out under forcecontrolled or displacement-controlled conditions. For force-controlled experiments it is $\xi=3$, and in the case of displacement-control, $\xi=1$. The relationships between the global contact quantities can also be formulated in a normalized form. For this purpose all quantities are normalized to their critical values:

$$
\hat{a}:=\frac{a}{a_{c}}, \quad \hat{d}:=\frac{d}{\left|d_{c}\right|}, \quad \hat{F}:=\frac{F_{N}}{\left|F_{c}\right|} .
$$

If we choose the critical values under force-controlled conditions, the normalized relationships are:

$$
\begin{aligned}
& \hat{d}(\hat{a})=3 \hat{a}-2 \sqrt{\hat{a}}, \\
& \hat{F}(\hat{a})=3 \hat{a}^{2}-4 \sqrt{\hat{a}^{3}} .
\end{aligned}
$$

These functions, $\hat{d}=\hat{d}(\hat{a})$ and $\hat{F}=\hat{F}(\hat{a})$, are shown in Fig. 3.3. The resulting, implicitly defined, dependency $\hat{F}=\hat{F}(\hat{d})$ is given in Fig. 3.4.

\subsubsection{The Paraboloid}

The adhesive contact problem for a parabolic body $\tilde{z}=r^{2} /(2 R)$ (see Fig. 3.5) was solved in the aforementioned classic JKR paper (Johnson et al. 1971). With the 
Fig. 3.3 The normalized normal force and indentation depth as functions of the normalized contact radius for adhesive indentation by a cone. All values are normalized to the critical state in the case of force-control

Fig. 3.4 Relationship between the normalized normal force and the normalized indentation depth for the adhesive indentation by a cone. All normalizations refer to the critical state in the case of force-control. The dashed part describes the states that are stable only for displacement-control
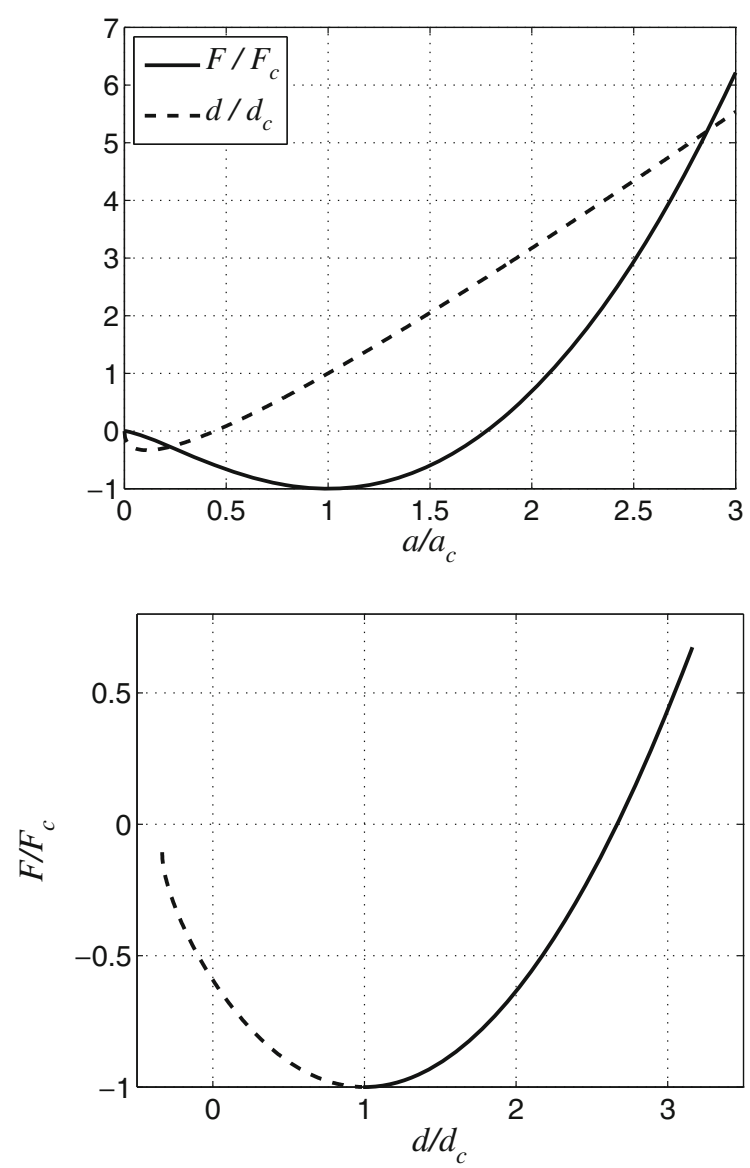

results from Chap. 2 (Sect. 2.5.3) and (3.18) and (3.20), we obtain:

$$
\begin{aligned}
d(a)= & \frac{a^{2}}{R}-\sqrt{\frac{2 \pi a \Delta \gamma}{E^{*}}}, \\
F_{N}(a)= & \frac{4}{3} \frac{E^{*} a^{3}}{R}-\sqrt{8 \pi a^{3} E^{*} \Delta \gamma}, \\
\sigma_{z z}(r ; a)= & -\frac{2 E^{*}}{\pi R} \sqrt{a^{2}-r^{2}}+\sqrt{\frac{2 E^{*} \Delta \gamma}{\pi a}} \frac{a}{\sqrt{a^{2}-r^{2}}}, \quad r \leq a, \\
w(r ; a)= & \frac{a^{2}}{\pi R}\left[\left(2-\frac{r^{2}}{a^{2}}\right) \arcsin \left(\frac{a}{r}\right)+\frac{\sqrt{r^{2}-a^{2}}}{a}\right]-\sqrt{\frac{8 a \Delta \gamma}{\pi E^{*}}} \arcsin \left(\frac{a}{r}\right), \\
& r>a .
\end{aligned}
$$


Fig. 3.5 Adhesive normal contact between a parabolic indenter and an elastic halfspace

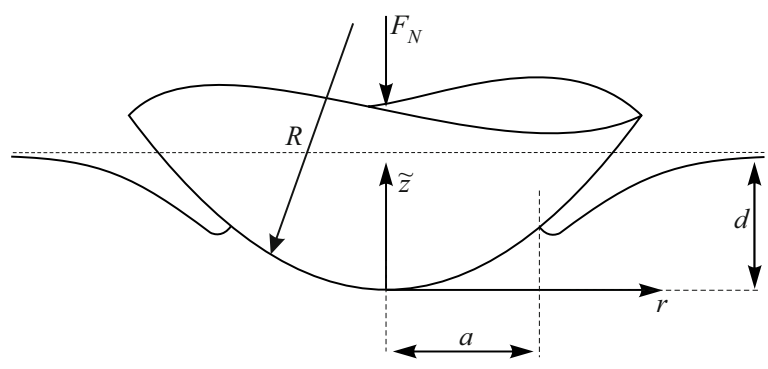

The critical contact radius and the corresponding values for the indentation depth and the normal force are given by (3.23):

$$
\begin{aligned}
& a_{c}=\left(\frac{\pi \xi^{2} R^{2} \Delta \gamma}{8 E^{*}}\right)^{1 / 3}, \\
& d_{c}=\left(\frac{\pi^{2} \xi(\Delta \gamma)^{2} R}{\left(E^{*}\right)^{2}}\right)^{1 / 3}\left(\frac{\xi}{4}-1\right), \\
& F_{c}=\pi \xi \Delta \gamma R\left(\frac{\xi}{6}-1\right),
\end{aligned}
$$

where $\xi=3$ for force-controlled trials and $\xi=1$ for displacement-controlled trials. In their explicit forms:

$$
\begin{aligned}
a_{c} & =\left(\frac{9 \pi R^{2} \Delta \gamma}{8 E^{*}}\right)^{1 / 3}, \\
d_{c} & =-\frac{1}{4}\left(\frac{3 \pi^{2}(\Delta \gamma)^{2} R}{\left(E^{*}\right)^{2}}\right)^{1 / 3}, \text { under force-control, } \\
F_{c} & =-\frac{3}{2} \pi \Delta \gamma R
\end{aligned}
$$

and

$$
\begin{aligned}
a_{c} & =\left(\frac{\pi R^{2} \Delta \gamma}{8 E^{*}}\right)^{1 / 3}, \\
d_{c} & =-\frac{3}{4}\left(\frac{\pi^{2}(\Delta \gamma)^{2} R}{\left(E^{*}\right)^{2}}\right)^{1 / 3}, \text { under displacement-control. } \\
F_{c} & =-\frac{5}{6} \pi \Delta \gamma R
\end{aligned}
$$

In this case, the critical force does not depend on $E^{*}$; i.e., it does not depend on the elastic properties of the half-space. In Sect. 3.5.7 it is demonstrated that the paraboloid is the only mnemonic indenter for which this is valid. After normalizing 
Fig. 3.6 The normalized normal force and indentation depth as functions of the contact radius for the adhesive indentation by a paraboloid. All values are normalized to the critical state under force-controlled boundary conditions

Fig. 3.7 Relationship between the normalized normal force and the normalized indentation depth for the adhesive indentation by a paraboloid. All normalizations are done with respect to the critical state under forcecontrol. The dotted section represents the states that are only stable for displacementcontrolled trials
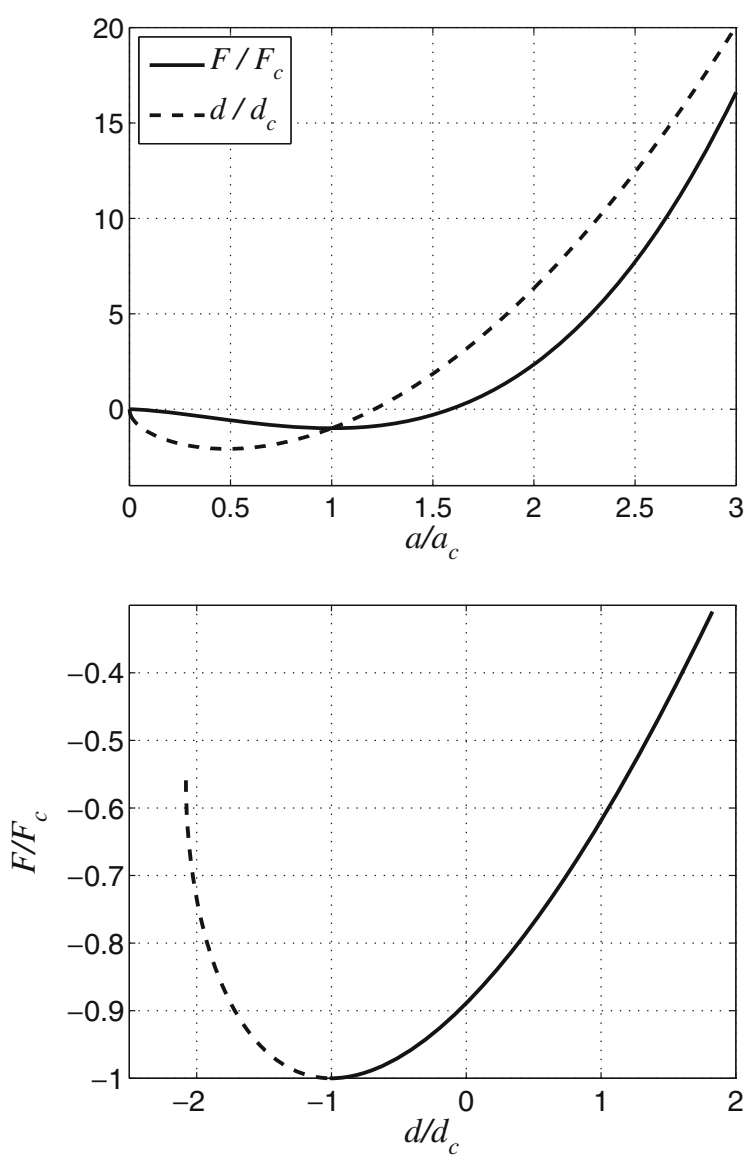

the quantities to the critical values of the force-controlled trial

$$
\hat{a}:=\frac{a}{a_{c}}, \quad \hat{d}:=\frac{d}{\left|d_{c}\right|}, \quad \hat{F}:=\frac{F_{N}}{\left|F_{c}\right|},
$$

the relationships $\left(3.44_{1}\right)$, and $\left(3.44_{2}\right)$, can be rewritten in a universal, dimensionless form:

$$
\begin{aligned}
& \hat{d}=3 \hat{a}^{2}-4 \sqrt{\hat{a}}, \\
& \hat{F}=\hat{a}^{3}-2 \sqrt{\hat{a}^{3}} .
\end{aligned}
$$

These relationships, $\hat{d}=\hat{d}(\hat{a})$ and $\hat{F}=\hat{F}(\hat{a})$, are illustrated in Fig. 3.6. The implicitly defined function $\hat{F}=\hat{F}(\hat{d})$ is given in Fig. 3.7.

\section{The Adhesive Impact Problem for the Parabolic Indenter}

The adhesive normal impact of a parabolic body has been heavily investigated due to its various technical applications. Thornton and Ning (1998) were able to analytically determine the coefficient of restitution for the JKR adhesive normal impact. 
Let the body have the mass $m$ and the initial velocity $v_{0}$. For the rebound velocity $v_{e}$ and the corresponding coefficient of restitution $e$, we obtain the expression:

$$
e:=\frac{v_{e}}{v_{0}}=\sqrt{1-\beta}, \quad \beta \leq 1,
$$

with

$$
\beta:=\frac{1}{5 m v_{0}^{2}}\left[\frac{R^{4}(\pi \Delta \gamma)^{5}}{E^{* 2}}\right]^{1 / 3}[1+\sqrt[3]{864}] .
$$

For $\beta>1$ the coefficient of restitution is zero, i.e., low initial velocities will cause the body to stick to the elastic half-space without rebounding.

\subsubsection{The Sphere}

It has already been discussed in Chap. 2 that this contact problem is very similar to the one described in the previous section. With the non-adhesive solution (see Sect. 2.5.4), and (3.18) and (3.20), we obtain the following solution to the adhesive contact problem:

$$
\begin{aligned}
d(a)= & a \operatorname{artanh}\left(\frac{a}{R}\right)-\sqrt{\frac{2 \pi a \Delta \gamma}{E^{*}}}, \\
F_{N}(a)= & E^{*} R^{2}\left[\left(1+\frac{a^{2}}{R^{2}}\right) \operatorname{artanh}\left(\frac{a}{R}\right)-\frac{a}{R}\right]-\sqrt{8 \pi a^{3} E^{*} \Delta \gamma} \\
\sigma_{z z}(r ; a)= & -\frac{E^{*}}{\pi}\left[\frac{R}{\sqrt{R^{2}-r^{2}}} \operatorname{artanh}\left(\frac{\sqrt{a^{2}-r^{2}}}{\sqrt{R^{2}-r^{2}}}\right)+\int_{r}^{a} \operatorname{artanh}\left(\frac{x}{R}\right) \frac{\mathrm{d} x}{\sqrt{x^{2}-r^{2}}}\right] \\
& +\sqrt{\frac{2 E^{*} \Delta \gamma}{\pi a} \frac{a}{\sqrt{a^{2}-r^{2}}}}, \quad r \leq a, \\
w(r ; a)= & w_{\text {n.a. }}(r ; a)-\sqrt{\frac{8 a \Delta \gamma}{\pi E^{*}}} \arcsin \left(\frac{a}{r}\right), \quad r>a
\end{aligned}
$$

with the sphere radius $R$. Here, $w_{\text {n.a. }}$ denotes the displacements without adhesion:

$$
\begin{aligned}
& w_{\text {n.a. }}(r ; a)=\frac{2}{\pi}\left\{\operatorname{artanh}\left(\frac{a}{R}\right)\left[a \arcsin \left(\frac{a}{r}\right)+\sqrt{r^{2}-a^{2}}\right]-R \arcsin \left(\frac{a}{r}\right)\right. \\
& \left.+\sqrt{R^{2}-r^{2}} \arctan \left(\frac{a \sqrt{R^{2}-r^{2}}}{R \sqrt{r^{2}-a^{2}}}\right)\right\} \text {. }
\end{aligned}
$$

The critical contact radius $a_{c}$, at which the contact loses its stability and detaches, is given by the numerical solution of the transcendental equation

$$
\operatorname{artanh} \alpha+\frac{\alpha}{1-\alpha^{2}}-\frac{\beta}{\sqrt{\alpha}}=0,
$$


a

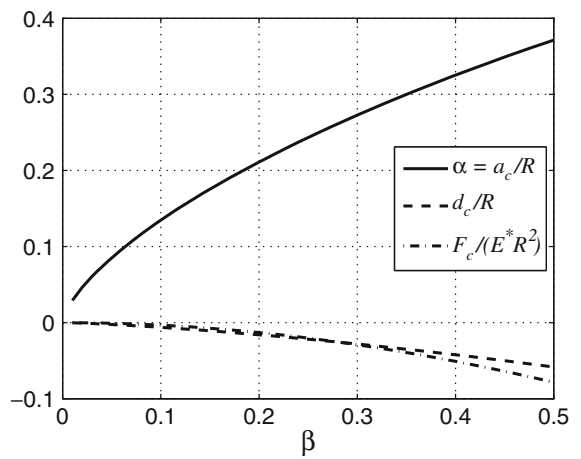

b

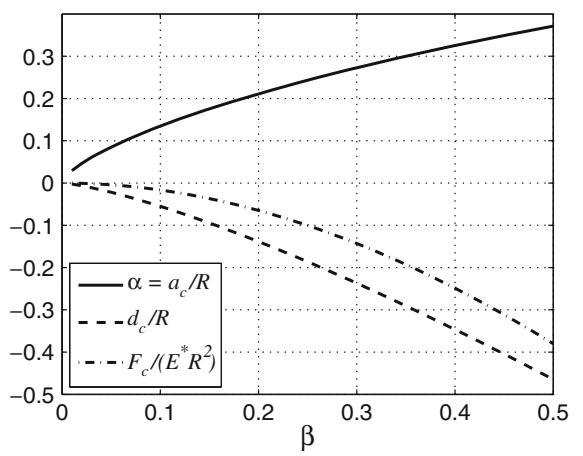

Fig. 3.8 Plots of the normalized critical contact radius $a_{c} / R$, the corresponding normalized indentation depth $d_{c} / R$, and normal force $F_{c} /\left(E^{*} R^{2}\right)$ as functions of the normalized surface energy $\beta$ (see (3.55)), according to (3.54) and (3.56); (a) force-control, (b) displacement-control

with $\alpha:=a_{c} / R$ and

$$
\beta:=\xi \sqrt{\frac{\pi \Delta \gamma}{2 E^{*} R}} .
$$

The last parameter describes a normalized surface energy and depends on the type of boundary condition ( $\xi=3$ for force-control, $\xi=1$ for displacement-control). For the relationships between the critical indentation depth, the critical contact radius, and the critical normal force, one then obtains the equations:

$$
\begin{aligned}
& d_{c}(\alpha)=R \alpha\left[\frac{\beta}{\sqrt{a}}\left(1-\frac{2}{\xi}\right)-\frac{\alpha}{1-\alpha^{2}}\right] \\
& F_{c}(\alpha)=E^{*} R^{2}\left[\frac{\beta}{\sqrt{a}}\left(1+\alpha^{2}-\frac{4 \alpha^{2}}{\xi}\right)-\frac{2 \alpha}{1-\alpha^{2}}\right] .
\end{aligned}
$$

These results for the critical values of contact radius, indentation depth, and normal force-in normalized form-are shown in Fig. 3.8. The lengths are normalized to the sphere radius and the "adhesion force" to $E^{*} R^{2}$. Since the half-space hypothesis is too severely violated for $a>0.3 R$, it can be seen from the diagram that these results are valid only for $\beta<0.35$. For example, it means that the effective surface energy in the case of force-control must not exceed $\Delta \gamma=9 \cdot 10^{4} \mathrm{~J} / \mathrm{m}^{2}$ for a sphere with $R=10^{-2} \mathrm{~m}$ and $E^{*}=10^{9} \mathrm{~Pa}$. That is an extremely great value though. In the case of displacement-control, this limit is even greater by a factor of 9 .

\subsubsection{The Ellipsoid}

It has been demonstrated in Chap. 2 (Sect. 2.5.5) that there is only a slight difference between the contact problems of an indenting sphere and an ellipsoid of rotation. 
The solution to the contact problem is, considering (3.18) and (3.20), given by:

$$
\begin{aligned}
d(a)= & a \operatorname{artanh}(k a)-\sqrt{\frac{2 \pi a \Delta \gamma}{E^{*}}}, \\
F_{N}(a)= & E^{*} \frac{R}{k}\left[\left(1+k^{2} a^{2}\right) \operatorname{artanh}(k a)-k a\right]-\sqrt{8 \pi a^{3} E^{*} \Delta \gamma}, \\
\sigma_{z z}(r ; a)= & -\frac{E^{*} k R}{\pi}\left[\frac{1}{\sqrt{1-k^{2} r^{2}}} \operatorname{artanh}\left(\frac{k \sqrt{a^{2}-r^{2}}}{\sqrt{1-k^{2} r^{2}}}\right)+\int_{r}^{a} \frac{\operatorname{artanh}(k x)}{\sqrt{x^{2}-r^{2}}} \mathrm{~d} x\right] \\
& +\sqrt{\frac{2 E^{*} \Delta \gamma}{\pi a} \frac{a}{\sqrt{a^{2}-r^{2}}}, \quad r \leq a,} \\
w(r ; a)= & k R w_{K, \text { n.a. }}\left(r ; a ; R=\frac{1}{k}\right)-\sqrt{\frac{8 a \Delta \gamma}{\pi E^{*}}} \arcsin \left(\frac{a}{r}\right), \quad r>a .
\end{aligned}
$$

Here, $k$ and $R$ denote the geometric parameters of the indenter profile which can be written as:

$$
f(r)=R\left(1-\sqrt{1-k^{2} r^{2}}\right) .
$$

$w_{K, \text { n.a. }}(r ; a ; R)$ is the displacement without adhesion for a spherical indenter of radius $R$, given in (3.53) of the previous section. The determining equation for the critical contact radius is again given by (3.54), wherein $\alpha:=k a_{c}$ and $\beta:=$ $\xi \sqrt{\frac{\pi \Delta \gamma}{2 E^{*} k R^{2}}}$. The expressions (3.56) for the critical values of indentation depth and normal force remain the same, except that the factor in front of the parenthesis in the normal force must be replaced by $E^{*} R / k$. It is therefore also possible to directly apply the curves in Fig. 3.8 since normalized curves are shown there.

\subsubsection{The Indenter Which Generates a Constant Adhesive Tensile Stress}

During their research on biological systems, in which adhesion played a central role (e.g., with a focus on geckos and certain insects), Gao and Yao (2004) came across the problem of the optimal (from a contact mechanical point of view) profile at the hair tips found on, for example, the gecko's feet, which is responsible for the strong adhesive forces in these systems. In this context, optimal means achieving the greatest possible pull-off force with the smallest possible contact area. To determine this optimal profile shape, Gao and Yao set out with a few preliminary considerations. Firstly, the critical state should correspond to a contact of the entire available contact domain. Secondly, the stress at the edges of the adhesive contact usually appears as a singularity since, at least according to the JKR theory, the adhesion itself can be interpreted as an indentation by a cylindrical flat punch. And since, ultimately, the maximum adhesive tension between two surfaces is solely determined by the potential of the van der Waals interaction between said surfaces, 
i.e., their material properties, the authors concluded that the optimal profile is the one which generates a constant adhesive tensile stress $\sigma_{0}$ in the contact area. The theoretical maximum pull-off force for a given contact area is then attributed to the profile where this stress $\sigma_{0}$ corresponds to the maximum adhesive stress $\sigma_{\text {th }}$.

The problem of the elastic half-space displacement resulting from the constant circular pressure or tension distribution of radius $a$ was previously considered in Chap. 2 (see Sect. 2.5.6). For a stress distribution

$$
\sigma_{z z}(r ; a)=\sigma_{0}, \quad r \leq a,
$$

the resulting displacement of the half-space is:

$$
w\left(r ; a, \sigma_{0}\right)=-\frac{4 \sigma_{0} a}{\pi E^{*}} \mathrm{E}\left(\frac{r}{a}\right), \quad r \leq a .
$$

This means that the optimal profile takes on the form:

$$
f\left(r ; a, \sigma_{0}\right)=\frac{\sigma_{0} a}{E^{*}}\left[\frac{4}{\pi} \mathrm{E}\left(\frac{r}{a}\right)-2\right], \quad r \leq a .
$$

Here, $\mathrm{E}(\cdot)$ denotes the complete elliptical integral of the second kind:

$$
\mathrm{E}(k):=\int_{0}^{\pi / 2} \sqrt{1-k^{2} \sin ^{2} \varphi} \mathrm{d} \varphi
$$

The pull-off force $F_{c}$ necessary to separate such an indenter profile from complete contact is trivially

$$
F_{c}=\pi \sigma_{0} a^{2} .
$$

The displacements of the half-space beyond the contact area were also previously calculated and equal

$$
w\left(r ; a, p_{0}\right)=\frac{4 \sigma_{0} r}{\pi E^{*}}\left[\left(1-\frac{a^{2}}{r^{2}}\right) \mathrm{K}\left(\frac{a}{r}\right)-\mathrm{E}\left(\frac{a}{r}\right)\right], \quad r>a,
$$

with the complete elliptical integral of the first kind:

$$
\mathrm{K}(k):=\int_{0}^{\pi / 2} \frac{\mathrm{d} \varphi}{\sqrt{1-k^{2} \sin ^{2} \varphi}} .
$$

The maximum pull-off force of this profile can be compared to the flat punch of identical radius $a$ :

$$
F_{\max }^{\text {Kendall }}=\sqrt{8 \pi a^{3} E^{*} \Delta \gamma}=\sqrt{8 \pi a^{3} E^{*} \sigma_{0} h} .
$$


For the optimal profile, the maximum force is:

$$
F_{\max }^{\mathrm{opt}}=\pi \sigma_{0} a^{2}
$$

Analogous to the theory of Maugis described in Sect. 3.8, the surface energy $\Delta \gamma$ is calculated from the adhesive stresses $\sigma_{t h}$ and the maximum range of the van der Waals interaction:

$$
\Delta \gamma=\sigma_{0} h
$$

The force ratio then equals:

$$
\frac{F_{\max }^{\text {Kendall }}}{F_{\max }^{\mathrm{opt}}}=\sqrt{\frac{8}{\pi} \frac{E^{*}}{\sigma_{0}} \frac{h}{a}} .
$$

\subsubsection{The Profile in the Form of a Power-Law}

We now consider a general indenter with the profile:

$$
f(r)=c r^{n}, \quad n \in \mathbb{R}^{+},
$$

with an arbitrary constant $c$ and a positive real exponent $n$. In contrast to the results for non-adhesive contact detailed in the previous chapter, for the adhesive contact, we obtain qualitatively different behavior for $n>0.5$ and $n<0.5$. We first turn our attention to the case of $n>0.5$. The contact problem was first investigated by Borodich and Galanov (2004), Spolenak et al. (2005), and Yao and Gao (2006). The solution of the contact problem shown in Fig. 3.9 is, as before, given by the solution of the non-adhesive contact (see Sect. 2.5.8) and (3.18) and (3.20):

$$
\begin{aligned}
d(a) & =\kappa(n) c a^{n}-\sqrt{\frac{2 \pi a \Delta \gamma}{E^{*}}}, \\
F_{N}(a) & =E^{*} \frac{2 n}{n+1} \kappa(n) c a^{n+1}-\sqrt{8 \pi a^{3} E^{*} \Delta \gamma}, \\
\sigma_{z z}(r ; a) & =-\frac{E^{*}}{\pi} n \kappa(n) c \int_{r}^{a} x^{n-1} \frac{\mathrm{d} x}{\sqrt{x^{2}-r^{2}}}+\sqrt{\frac{2 E^{*} \Delta \gamma}{\pi a}} \frac{a}{\sqrt{a^{2}-r^{2}}}, \quad r \leq a, \\
w(r ; a) & =\frac{2}{\pi} \kappa(n) c\left[a^{n} \arcsin \left(\frac{a}{r}\right)-\int_{0}^{a} x^{n} \frac{\mathrm{d} x}{\sqrt{r^{2}-x^{2}}}\right]-\sqrt{\frac{8 a \Delta \gamma}{\pi E^{*}}} \arcsin \left(\frac{a}{r}\right), \\
r>a . &
\end{aligned}
$$

Similarly to Chap. 2, we introduce the scaling factor:

$$
\kappa(n):=\sqrt{\pi} \frac{\Gamma(n / 2+1)}{\Gamma[(n+1) / 2]},
$$


Fig. 3.9 Adhesive normal contact between a rigid indenter with a profile in the form of a power-law and an elastic half-space

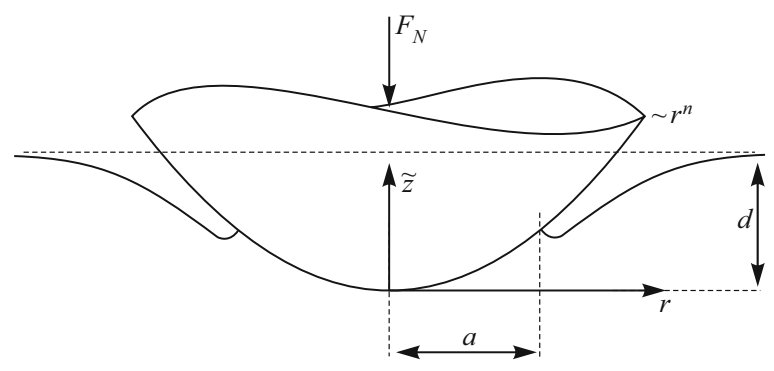

with the gamma function $\Gamma(\cdot)$ :

$$
\Gamma(z):=\int_{0}^{\infty} t^{z-1} \exp (-t) \mathrm{d} t .
$$

With regard to the different possibilities of the resolution of the integrals occurring in the stresses and displacements see Sect. 2.5.8, which details the consideration of power profiles. For the critical contact radius, we obtain this with (3.23):

$$
a_{c}=\left(\frac{\xi^{2} \pi \Delta \gamma}{2 E^{*} n^{2} c^{2} \kappa^{2}(n)}\right)^{\frac{1}{2 n-1}} .
$$

The critical indentation depth is:

$$
d_{c}=\left(\frac{\pi \Delta \gamma}{2 E^{*}}\right)^{\frac{n}{2 n-1}}\left(\frac{\xi}{n c \kappa(n)}\right)^{\frac{1}{2 n-1}}\left[\frac{\xi}{n}-2\right] .
$$

It is positive in the force-controlled case $(\xi=3)$ if $n<1.5$. Under displacementcontrol it is $\xi=1$. The "adhesion force" is given by:

$$
F_{c}=\left(E^{*}\right)^{\frac{n-2}{2 n-1}}\left(\frac{\pi \Delta \gamma}{2}\right)^{\frac{n+1}{2 n-1}}\left(\frac{\xi}{n c \kappa(n)}\right)^{\frac{3}{2 n-1}}\left[\frac{2 \xi}{n+1}-4\right] .
$$

It can be seen that the Hertzian contact for $n=2$ is the only case in which this force does not depend on $E^{*}$. By introducing the quantities

$$
\hat{a}:=\frac{a}{a_{c}}, \quad \hat{d}:=\frac{d}{\left|d_{c}\right|}, \quad \hat{F}:=\frac{F_{N}}{\left|F_{c}\right|},
$$

normalized to the critical values, the relations between the macroscopic quantities can be written as:

$$
\begin{aligned}
& \hat{d}=\frac{\xi}{|\xi-2 n|} \hat{a}^{n}-\frac{2 n}{|\xi-2 n|} \sqrt{\hat{a}} \\
& \hat{F}=\frac{\xi}{|\xi-2 n-2|} \hat{a}^{n+1}-\frac{2 n+2}{|\xi-2 n-2|} \sqrt{\hat{a}^{3}} .
\end{aligned}
$$


Fig. 3.10 Normalized indentation depth as a function of the normalized contact radius for an indenter with a profile in the shape of a powerlaw with exponent $n$. All quantities are normalized by the critical values in the force-controlled experiment. Shown are different values of $n$

Fig. 3.11 Normalized normal force as a function of the normalized contact radius for an indenter with a profile in the shape of a power-law with exponent $n$. All quantities are normalized by the critical values in the force-controlled experiment. Shown are different values of $n$
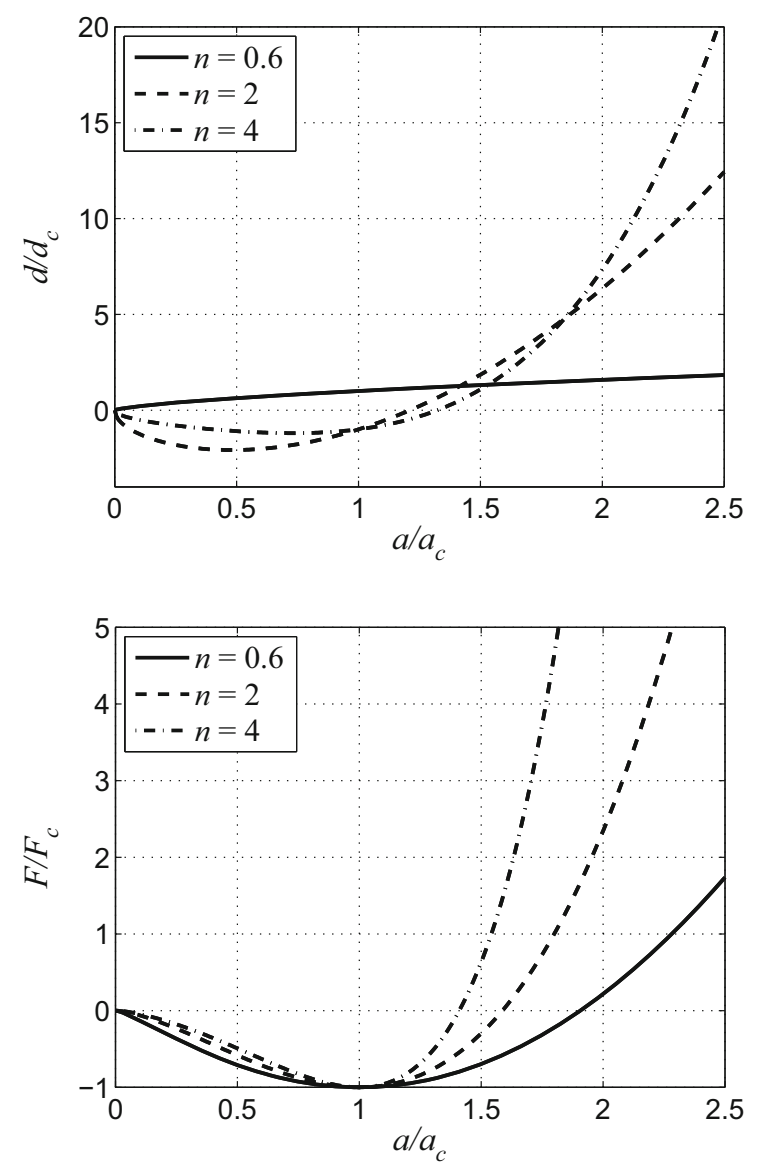

It is interesting to note that the relations (3.78), except for the type of boundary condition (force- or displacement-control), only depend on the exponent $n$. The relationships (3.78) are shown for the force-controlled experiment in Figs. 3.10 and 3.11 for three different values of $n$.

All of the aforementioned results also apply to indenters with $n<1 / 2$ (Popov 2017). What changes is just the interpretation of the corresponding quantities. In the case of $n>1 / 2$ the critical quantities separate the state of stable adhesive contact with a finite contact radius from the process of unstable shrinkage of the contact area and complete detachment. A stable condition exists with larger forces and an unstable condition with smaller forces. On the other hand, in the case of $n<1 / 2$ the critical quantities separate the stable state from the unstable, unlimited propagation of the contact area. The stable state exists for smaller forces and the instability occurs when increasing the force (or indentation). A detailed analysis was given by Popov (2017). 
A special case is $n=0.5$. We want to discuss this case under the condition of a displacement-controlled loading. It is, in this case:

$$
\begin{aligned}
g(a) & =\kappa(1 / 2) c a^{1 / 2}, \quad \text { with } \quad \kappa(1 / 2)=\frac{1}{4} \frac{\pi^{3 / 2} \sqrt{2}}{\Gamma(3 / 4)^{2}} \approx 1.311, \\
\Delta l(a) & =\left(\frac{2 \pi \Delta \gamma}{E^{*}}\right)^{1 / 2} a^{1 / 2} .
\end{aligned}
$$

At the moment of the first contact, $d=0$, it applies to all $a$ :

$$
\begin{gathered}
\text { I. } g(a)>\Delta l(a), \quad \text { if } c>1.9120\left(\frac{\Delta \gamma}{E^{*}}\right)^{1 / 2}, \\
\text { II. } g(a)<\Delta l(a), \quad \text { if } c<1.9120\left(\frac{\Delta \gamma}{E^{*}}\right)^{1 / 2} \text {. }
\end{gathered}
$$

In the first case, the radius of the contact will decrease until it disappears. In the second case, it will enlarge until complete contact is established. Furthermore, in the second case, complete contact is formed immediately, as soon as the indenter tip touches the half-space.

\section{The Adhesive Impact Problem for the Indenter with Power-Law Profile}

The normal adhesive impact problem, as in the case of the parabolic body, can be solved in general form. The body has the mass $m$ and the initial velocity $v_{0}$. For the rebound speed $v_{e}$, and thus the coefficient of restitution $e$, we obtain:

$$
e:=\frac{v_{e}}{v_{0}}=\sqrt{1-\beta}, \quad \beta \leq 1
$$

with

$$
\begin{aligned}
\beta:= & \frac{1}{m v_{0}^{2}}\left[\left(\frac{\pi \Delta \gamma}{2}\right)^{2 n+1}\left(\frac{1}{E^{*} n^{2} c^{2} \kappa^{2}(n)}\right)^{2}\right]^{\frac{1}{2 n-1}} \\
& \cdot \frac{8 n-4}{2 n^{2}+3 n+1}\left[n+(n+1)(2 n)^{\frac{4}{2 n-1}}\right] .
\end{aligned}
$$

For $\beta>1$ it is $e=0$; that is, the body will stick to the elastic half-space and not rebound for impact velocities below a critical value. For $n=2$, the known solution from Sect. 3.5.3 is recovered.

\subsubsection{The Truncated Cone}

The adhesive normal contact problem for a truncated cone (see Fig. 3.12) was first solved by Maugis and Barquins (1983). With the help of the solutions from Chap. 2 
Fig. 3.12 Adhesive normal contact between a rigid truncated cone and an elastic half-space

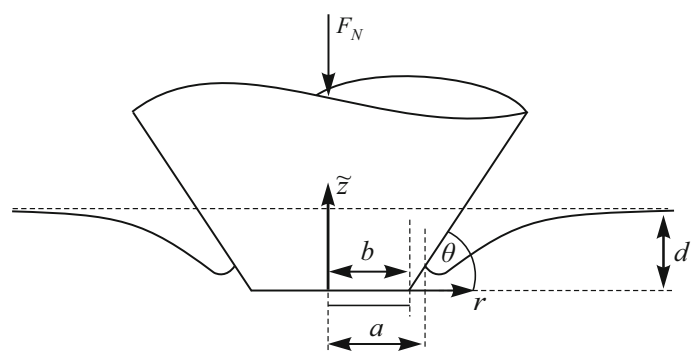

(see Sect. 2.5.9) and (3.18) and (3.20), the solution of the adhesive normal contact problem can be determined without difficulty:

$$
\begin{aligned}
d(a) & =a \tan \theta \arccos \left(\frac{b}{a}\right)-\sqrt{\frac{2 \pi a \Delta \gamma}{E^{*}}}, \\
F_{N}(a) & =E^{*} \tan \theta a^{2}\left[\arccos \left(\frac{b}{a}\right)+\frac{b}{a} \sqrt{1-\frac{b^{2}}{a^{2}}}\right]-\sqrt{8 \pi a^{3} E^{*} \Delta \gamma}, \\
\sigma_{z z}(r ; a) & =\sigma_{z z \text {,n.a. }}(r ; a)+\sqrt{\frac{2 E^{*} \Delta \gamma}{\pi a}} \frac{a}{\sqrt{a^{2}-r^{2}}}, \quad r \leq a, \\
w(r ; a) & =w_{\text {n.a. }}(r ; a)-\sqrt{\frac{8 a \Delta \gamma}{\pi E^{*}}} \arcsin \left(\frac{a}{r}\right), \quad r>a .
\end{aligned}
$$

Here, $b$ denotes the radius at the blunt end and $\theta$ the conical slope angle. The solutions of the stresses and displacements in the case of non-adhesive contact indicated by the index "n.a." can be looked up in Sect. 2.5.9:

$$
\begin{gathered}
\sigma_{z z, \text { n.a. }}(r ; a)= \\
-\frac{E^{*} \tan \theta}{\pi}\left\{\begin{array}{l}
\int_{b}^{a}\left\{\frac{b}{\sqrt{x^{2}-b^{2}}}+\arccos \left(\frac{b}{x}\right)\right\} \frac{\mathrm{d} x}{\sqrt{x^{2}-r^{2}}}, \quad r \leq b, \\
\int_{r}^{a}\left\{\frac{b}{\sqrt{x^{2}-b^{2}}}+\arccos \left(\frac{b}{x}\right)\right\} \frac{\mathrm{d} x}{\sqrt{x^{2}-r^{2}}}, \quad b<r \leq a, \\
w_{\text {n.a. }}(r ; a)=\frac{2 \tan \theta}{\pi}\left\{\varphi_{0} a \arcsin \left(\frac{a}{r}\right)-\int_{b}^{a} x \arccos \left(\frac{b}{x}\right) \frac{\mathrm{d} x}{\sqrt{r^{2}-x^{2}}}\right\}, \\
r>a .
\end{array}\right.
\end{gathered}
$$

The relationship between indentation depth and contact radius in the case without adhesion is described by:

$$
d_{\text {n.a. }}(a)=\varphi_{0} a \tan \theta,
$$

with:

$$
\varphi_{0}:=\arccos \left(\frac{b}{a}\right) .
$$


Fig. 3.13 Stability bifurcation for the angle $\varphi_{0}$ as a function of the normalized surface energy $\beta$ (see (3.88)) for the adhesive indentation by a truncated cone. All points between the two curves indicate unstable configurations

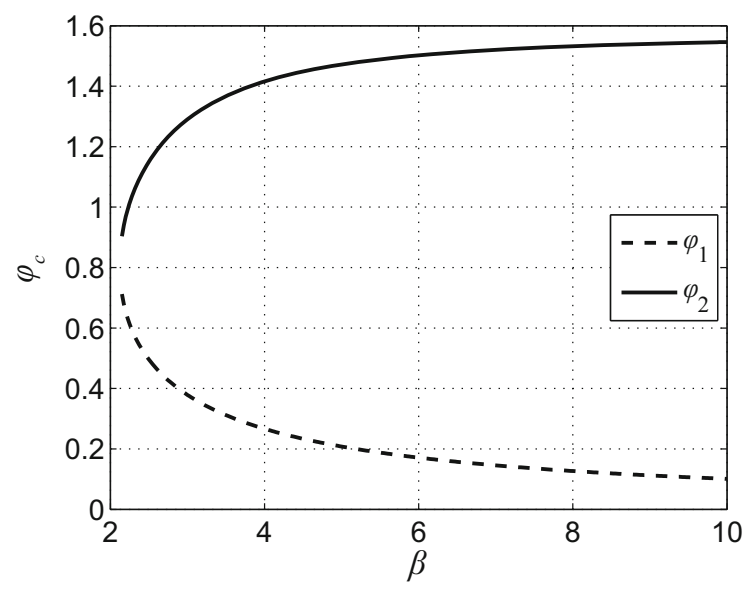

This results in the critical contact radius as a solution to the transcendental equation:

$$
\varphi_{0, c}+\cot \varphi_{0, c}-\beta \sqrt{\cos \varphi_{0 . c}}=0,
$$

with:

$$
\beta:=\frac{\xi}{\tan \theta} \sqrt{\frac{\pi \Delta \gamma}{2 E^{*} b}} .
$$

For the critical values of the indentation depth and the normal force, one obtains:

$$
\begin{aligned}
& d_{c}=a_{c} \tan \theta\left[\varphi_{0, c}\left(1-\frac{2}{\xi}\right)-\frac{2}{\xi} \cot \varphi_{0, c}\right], \\
& F_{c}=E^{*} \tan \theta a_{c}^{2}\left[\varphi_{0, c}\left(1-\frac{4}{\xi}\right)+\cot \varphi_{0, c}\left(\sin ^{2} \varphi_{0, c}-\frac{4}{\xi}\right)\right] .
\end{aligned}
$$

In (3.88) and (3.89), the type of boundary condition must yet be determined. Displacement-controlled trials are characterized by $\xi=1$, and force-controlled ones by $\xi=3$. One can easily convince oneself that for $b=0$, and thus $\varphi_{0} \equiv \pi / 2$, the solutions of the complete cone from Sect. 3.5.2 are recovered.

However, a closer look at (3.87) reveals a bifurcation of the solution. This is shown in Fig. 3.13. The equation only has solutions for $\beta>2.125$. For smaller values of $\beta$ the critical radius $a_{c}$ is given by the radius $b$ and the critical values of the indentation depth and normal force correspond to those of the flat punch:

$$
\begin{aligned}
& d_{c}=-\sqrt{\frac{2 \pi b \Delta \gamma}{E^{*}}}, \\
& F_{c}=-\sqrt{8 \pi b^{3} E^{*} \Delta \gamma} .
\end{aligned}
$$


Fig. 3.14 Solution for the normalized critical values for the contact radius $a_{c} / b$, indentation depth $d_{c} /(b \tan \theta)$, and the normal force $F_{c} /\left(2 E^{*} b^{2} \tan \theta\right)$ as a function of $\beta$ for the adhesive indentation by a truncated cone for the critical angle $\varphi_{1}$ under force-controlled conditions

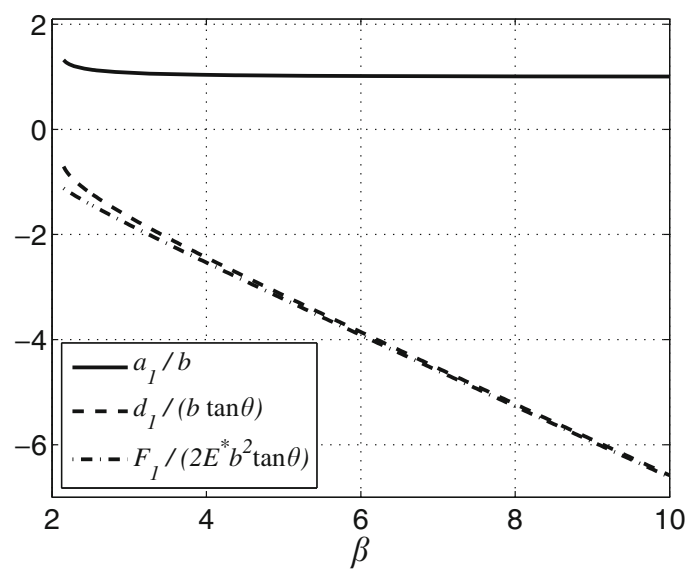

For $\beta>2.125$ there are always two roots of the equation and one can show that all configurations between these two roots (that is, all values $\varphi_{0, c 1}<\varphi_{0}<\varphi_{0, c 2}$ ) are unstable. For example, for $b=10^{-2} \mathrm{~m}, \theta=0.1, E^{*}=10^{9} \mathrm{~Pa}, \beta>2.125$ means that the effective surface energy must (in the force-controlled case) be $\Delta \gamma>$ $3 \cdot 10^{4} \mathrm{~J} / \mathrm{m}^{2}$. This is a very big value. In this case, the two solutions for the angle $\varphi_{0}$ are shown in Fig. 3.13. Figure 3.14 shows the curves of the contact radius, the indentation depth, and the normal force in normalized form for the first critical solution under force-control as a function of the normalized surface energy $\beta$.

\subsubsection{The Truncated Paraboloid}

With the results of Sect. 2.5.10 and (3.18) and (3.20), we come to the following solution first found by Maugis and Barquins (1983) regarding the adhesive normal contact problem for the truncated paraboloid (see Fig. 3.15):

$$
\begin{aligned}
d(a) & =\frac{a}{R} \sqrt{a^{2}-b^{2}}-\sqrt{\frac{2 \pi a \Delta \gamma}{E^{*}}}, \\
F_{N}(a) & =\frac{2 E^{*}}{3 R}\left(2 a^{2}+b^{2}\right) \sqrt{a^{2}-b^{2}}-\sqrt{8 \pi a^{3} E^{*} \Delta \gamma}, \\
\sigma_{z z}(r ; a) & =\sigma_{z z, \text { n.a. }}(r ; a)+\sqrt{\frac{2 E^{*} \Delta \gamma}{\pi a}} \frac{a}{\sqrt{a^{2}-r^{2}}}, \quad r \leq a, \\
w(r ; a) & =w_{\text {n.a. }}(r ; a)-\sqrt{\frac{8 a \Delta \gamma}{\pi E^{*}}} \arcsin \left(\frac{a}{r}\right), \quad r>a .
\end{aligned}
$$

Here, $b$ denotes the radius at the flat tip. The base paraboloid has the radius of curvature $R$. The solutions for the stresses and displacements in the non-adhesive 
Fig. 3.15 Adhesive normal contact between a rigid truncated paraboloid and an elastic half-space

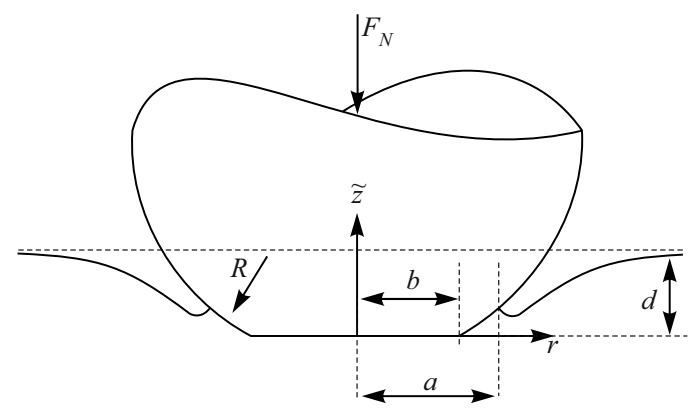

case, characterized by the index "n.a.", are:

$$
\begin{aligned}
& \sigma_{z z, \text { n.a. }}(r ; a)=-\frac{E^{*}}{\pi R} \begin{cases}\int_{b}^{a} \frac{\left(2 x^{2}-b^{2}\right) \mathrm{d} x}{\sqrt{x^{2}-b^{2}} \sqrt{x^{2}-r^{2}}}, & r \leq b, \\
\int_{r}^{a} \frac{\left(2 x^{2}-b^{2}\right) \mathrm{d} x}{\sqrt{x^{2}-b^{2}} \sqrt{x^{2}-r^{2}}}, & b<r \leq a,\end{cases} \\
& w_{\text {n.a. }}(r ; a)=\frac{2 a}{\pi R} \sqrt{a^{2}-b^{2}} \arcsin \left(\frac{a}{r}\right) \\
& -\frac{1}{\pi R}\left[\left(r^{2}-b^{2}\right) \arcsin \left(\frac{\sqrt{a^{2}-b^{2}}}{\sqrt{r^{2}-b^{2}}}\right)-\sqrt{a^{2}-b^{2}} \sqrt{r^{2}-a^{2}}\right], \\
& r>a \text {. }
\end{aligned}
$$

Between indentation depth and contact radius in the case of non-adhesive contact, the following relationship applies:

$$
d_{\text {n.a. }}(a)=\frac{a}{R} \sqrt{a^{2}-b^{2}}=\frac{a^{2}}{R} \sin \varphi_{0},
$$

with

$$
\varphi_{0}:=\arccos \left(\frac{b}{a}\right) .
$$

Equation (3.23) then provides the transcendental equation that determines the critical contact radius:

$$
\frac{1+\sin ^{2} \varphi_{0, c}}{\sin \varphi_{0, c} \cos \varphi_{0, c}}-\beta \sqrt{\cos \varphi_{0, c}}=0,
$$

with

$$
\beta:=\frac{\xi R}{b} \sqrt{\frac{\pi \Delta \gamma}{2 E^{*} b}} .
$$


Fig. 3.16 Stability bifurcation for the angle $\varphi_{0}$ as a function of the normalized surface energy $\beta$ (see (3.96)) for the adhesive indentation by a truncated paraboloid. All points between the two curves indicate unstable configurations

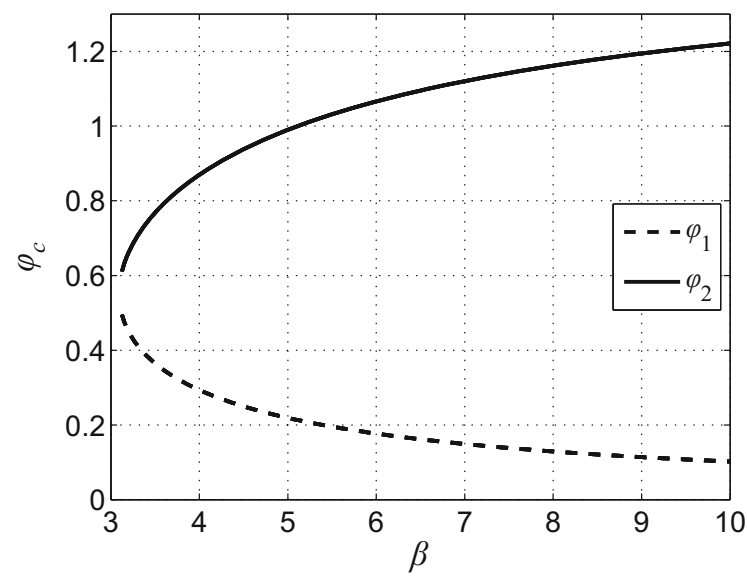

For the critical indentation depth and normal force, we obtain:

$$
\begin{aligned}
& d_{c}=\frac{a_{c}^{2}}{R} \sin \varphi_{0, c}\left(1-\frac{2}{\xi}-\frac{2}{\xi \sin ^{2} \varphi_{0, c}}\right), \\
& F_{c}=\frac{2 E^{*} a_{c}^{3}}{3 R} \sin \varphi_{0, c}\left[3-\sin ^{2} \varphi_{0, c}-\frac{6}{\xi}\left(1+\frac{1}{\sin ^{2} \varphi_{0, c}}\right)\right],
\end{aligned}
$$

which can be reduced to the results in Sect. 3.5.3 without great difficulty for $b=0$. In (3.95) and (3.97), the type of boundary condition has to be defined ( $\xi=1$ for displacement-control, $\xi=3$ for force-control).

Examining (3.95), one encounters a similar bifurcation as in the previous section. The equation only has solutions for $\beta>3.095$. For smaller values of $\beta$ the critical contact radius is given by $b$ and the associated values of indentation depth and normal force are the same as those of the flat punch. For a sufficiently large surface energy and, correspondingly, $\beta>3.095$, there are always two solutions to (3.95), and one finds that all states between these two solutions are unstable. These solutions as a function of $\beta$ are shown in Fig. 3.16. For $b=10^{-3} \mathrm{~m}, R=10^{-2} \mathrm{~m}$, $E^{*}=10^{9} \mathrm{~Pa}, \beta>3.095$ means (as an example) that the effective surface energy in the force-controlled case must be $\Delta \gamma>7 \cdot 10^{3} \mathrm{~J} / \mathrm{m}^{2}$. Figure 3.17 shows the curves of the contact radius, the indentation depth, and the normal force in normalized form for the first critical solution under force-control as a function of the normalized surface energy $\beta$.

\subsubsection{The Cylindrical Flat Punch with Parabolic Cap}

Consider now the adhesive normal contact between an elastic half-space and a flat punch with a parabolic cap. The punch has the radius $b$ and the cap has the radius of curvature $R$. 
Fig. 3.17 Dependencies of the normalized critical values for the contact radius $a_{c} / b$, the indentation depth $d_{c} R / b^{2}$, and the normal force $F_{c} R /\left(2 E^{*} b^{3}\right)$ as function of $\beta$ for the adhesive indentation by a truncated paraboloid at force-control for the critical angle $\varphi_{1}$

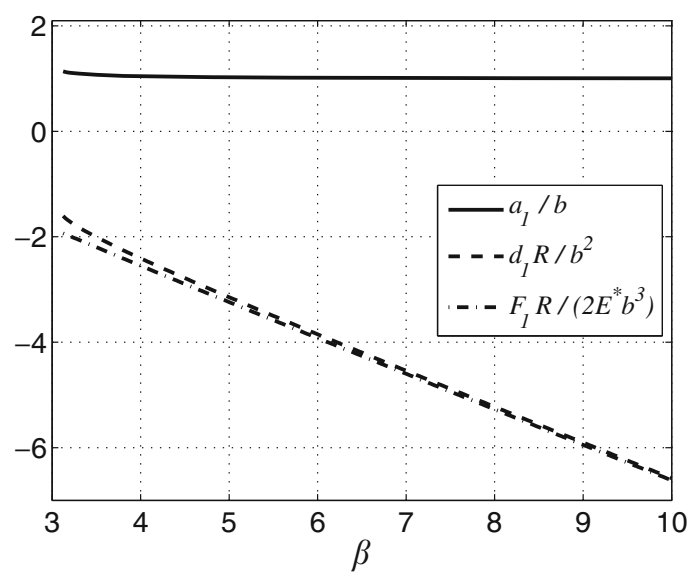

There may be two outcomes based on these examples. If the contact radius $a$ is smaller than $b$, the contact assembles simply the one with a parabolic indenter, for which the solution can be looked up in Sect. 3.5.3. With a sufficiently large indentation depth, or normal force, the contact radius is $a=b$. In this case, however, analogous to the problem of the flat punch, there is no difference between the equations of the non-adhesive and the adhesive solution. In Chap. 2 (Sect. 2.5.11), this distinction was not considered further because it had no appreciable consequences. In adhesive contact, however, this results in peculiarities regarding the stability of the contact. We therefore once again repeat the two aforementioned solutions. If $a<b$ is the solution of the contact problem, according to (3.18) and (3.20), it is as follows:

$$
\begin{aligned}
d(a)= & \frac{a^{2}}{R}-\sqrt{\frac{2 \pi a \Delta \gamma}{E^{*}},} \\
F_{N}(a)= & \frac{4}{3} \frac{E^{*} a^{3}}{R}-\sqrt{8 \pi a^{3} E^{*} \Delta \gamma}, \\
\sigma_{z z}(r ; a)= & -\frac{2 E^{*}}{\pi R} \sqrt{a^{2}-r^{2}}+\sqrt{\frac{2 E^{*} \Delta \gamma}{\pi a}} \frac{a}{\sqrt{a^{2}-r^{2}}}, \quad r \leq a, \\
w(r ; a)= & \frac{a^{2}}{\pi R}\left[\left(2-\frac{r^{2}}{a^{2}}\right) \arcsin \left(\frac{a}{r}\right)+\frac{\sqrt{r^{2}-a^{2}}}{a}\right] \\
& -\sqrt{\frac{8 a \Delta \gamma}{\pi E^{*}}} \arcsin \left(\frac{a}{r}\right), \quad r>a .
\end{aligned}
$$


In contrast, if $a=b$ the solution is described by:

$$
\begin{aligned}
F_{N}(d) & =2 E^{*}\left(d b-\frac{b^{3}}{3 R}\right), \\
\sigma_{z z}(r ; d) & =-\frac{E^{*}}{\pi R} \frac{b^{2}-2 r^{2}+d R}{\sqrt{b^{2}-r^{2}}}, \quad r \leq b, \\
w(r ; d) & =\frac{1}{\pi R}\left\{\left(2 d R-r^{2}\right) \arcsin \left(\frac{b}{r}\right)+b \sqrt{r^{2}-b^{2}}\right\}, \quad r>b .
\end{aligned}
$$

Depending on the value of the surface energy, different variants of the critical state are possible. If we denote the critical contact radius under force-controlled conditions with $a_{c}$ and those with displacement-control with $a_{c, d}$, these cases can be structured as follows:

- $\Delta \gamma<\Delta \gamma_{1}: a_{c}=a_{c}^{\mathrm{JKR}}, a_{c, d}=a_{c . d}^{\mathrm{JKR}}$

- $\Delta \gamma_{1} \leq \Delta \gamma<\Delta \gamma_{2}: a_{c}=b, a_{c, d} \stackrel{c . d}{=} a_{c . d}^{\mathrm{JKR}}$

- $\Delta \gamma>\Delta \gamma_{2}: a_{c}=a_{c, d}=b$

Here, the superscripts "JKR" denote the respective results for the parabolic indenter. $\Delta \gamma_{1}$ and $\Delta \gamma_{2}$ indicate the values of the surface energy at which the critical radii of the parabolic solution just coincide with the radius of the punch:

$$
\begin{aligned}
& a_{c}^{\mathrm{JKR}}\left(\Delta \gamma=\Delta \gamma_{1}\right)=b, \\
& a_{c, d}^{\mathrm{JKR}}\left(\Delta \gamma=\Delta \gamma_{2}\right)=b .
\end{aligned}
$$

From (3.100) one obtains (with (3.45)):

$$
\Delta \gamma_{2}=9 \Delta \gamma_{1}=\frac{8 E^{*} b^{3}}{\pi R^{2}} .
$$

By introducing the normalized quantities

$$
\hat{d}:=\frac{d}{\left|d_{c}^{\mathrm{JKR}}\right|}, \quad \hat{a}:=\frac{a}{a_{c}^{\mathrm{JKR}}}, \quad \hat{b}:=\frac{b}{a_{c}^{\mathrm{JKR}}}, \quad \hat{F}:=\frac{F_{N}}{\left|F_{c}^{\mathrm{JKR}}\right|},
$$

with the known results for the parabolic indenter (see Sect. 3.5.3),

$$
\begin{gathered}
a_{c}^{\mathrm{JKR}}=\left(\frac{9 \pi R^{2} \Delta \gamma}{8 E^{*}}\right)^{1 / 3}, \quad d_{c}^{\mathrm{JKR}}=-\frac{1}{4}\left(\frac{3 \pi^{2}(\Delta \gamma)^{2} R}{\left(E^{*}\right)^{2}}\right)^{1 / 3}, \\
F_{c}^{\mathrm{JKR}}=-\frac{3}{2} \pi \Delta \gamma R,
\end{gathered}
$$

the relationships between the global contact quantities can be written as follows:

$$
\begin{aligned}
& \hat{d}=3 \hat{a}^{2}-4 \sqrt{\hat{a}}, \\
& \hat{F}= \begin{cases}\hat{a}^{3}-2 \sqrt{\hat{a}^{3}}, & \hat{a}<\hat{b}, \\
\frac{1}{2}\left(\hat{d} \hat{b}-\hat{b}^{3}\right), & \hat{a}=\hat{b} .\end{cases}
\end{aligned}
$$




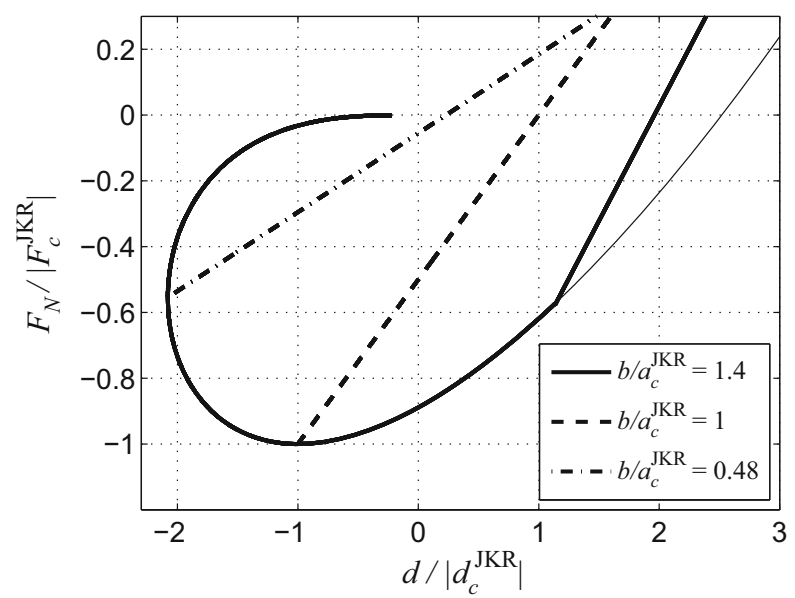

Fig. 3.18 Normalized relationship between normal force and indentation depth for a flat punch with parabolic cap at different normalized punch radii. The thin solid line corresponds to the parabolic JKR-solution

Thus the defined relationship $\hat{F}=\hat{F}(\hat{d})$ is shown in Fig. 3.18 for three different values of $\hat{b}$. Here, $\hat{b}=1$ corresponds to a surface energy of $\Delta \gamma=\Delta \gamma_{1}$ and $\hat{b}=0.48$ of a surface energy of $\Delta \gamma=\Delta \gamma_{2}$. It is easy to see the corresponding detachment points from the JKR solution for the paraboloid.

\subsubsection{The Cone with Parabolic Cap}

In Chap. 2 (see Sect. 2.5.12), the solution to the contact problem of a non-adhesive, frictionless, normal contact between an elastic half-space and a rigid cone with a rounded tip was shown. Thus, the solution of the adhesive, frictionless normal contact (see Fig. 3.19) is already known. The solution was first published by Maugis and Barquins (1983). With (3.18) and (3.20), one obtains:

$$
\begin{gathered}
d(a)=a \tan \theta\left(\frac{1-\sin \varphi_{0}}{\cos \varphi_{0}}+\varphi_{0}\right)-\sqrt{\frac{2 \pi a \Delta \gamma}{E^{*}}}, \\
F_{N}(a)=E^{*} a^{2} \tan \theta\left(\varphi_{0}+\frac{4}{3} \frac{1-\sin \varphi_{0}}{\cos \varphi_{0}}+\frac{1}{3} \sin \varphi_{0} \cos \varphi_{0}\right)-\sqrt{8 \pi a^{3} E^{*} \Delta \gamma}, \\
\sigma_{z z}(r ; a)=\sigma_{z z \text {.n.a. }}(r ; a)+\sqrt{\frac{2 E^{*} \Delta \gamma}{\pi a}} \frac{a}{\sqrt{a^{2}-r^{2}}}, \quad r \leq a, \\
w(r ; a)=w_{\text {n.a. }}(r ; a)-\sqrt{\frac{8 a \Delta \gamma}{\pi E^{*}}} \arcsin \left(\frac{a}{r}\right), \quad r>a,
\end{gathered}
$$


Fig. 3.19 Adhesive normal contact between a rigid cone with parabolic cap and an elastic half-space

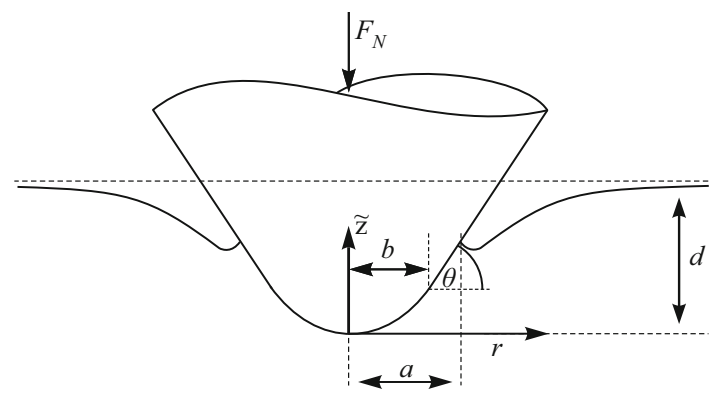

with

$$
\varphi_{0}:=\arccos \left(\frac{b}{a}\right),
$$

where $b$ denotes the radius at which the parabolic tip, which is continuously differentiable, passes into the conical body. $\theta$ describes the slope angle of the conical body. The stresses and displacements in the non-adhesive case, denoted by the index "n.a.", are given by:

$$
\begin{aligned}
& \sigma_{z z \text {,n.a. }}(r ; a)=
\end{aligned}
$$

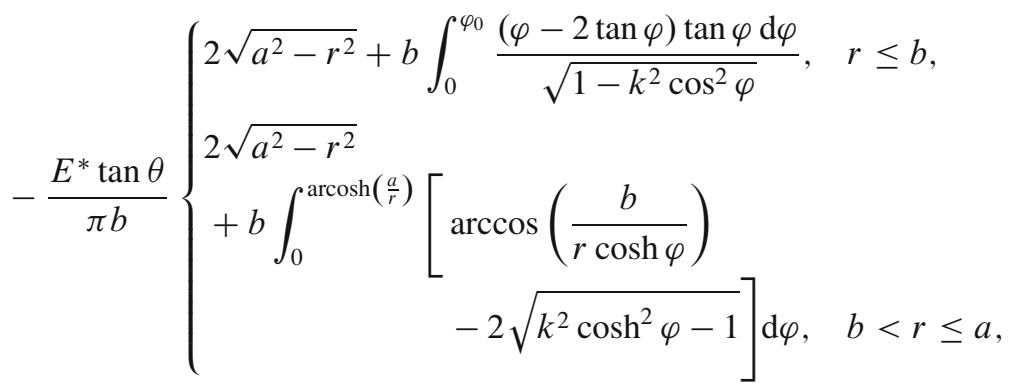

$$
\begin{aligned}
& w_{\text {n.a. }}(r ; a)=\frac{2 d_{\text {n.a. }}(a)}{\pi} \arcsin \left(\frac{a}{r}\right) \\
& -\frac{\tan \theta}{\pi b}\left[r^{2} \arcsin \left(\frac{a}{r}\right)\right. \\
& \left.-a \sqrt{r^{2}-a^{2}}+2 b^{2} \int_{0}^{\varphi_{0}} \frac{(\varphi-\tan \varphi) \tan \varphi \mathrm{d} \varphi}{\cos \varphi \sqrt{k^{2} \cos ^{2} \varphi-1}}\right], \quad r>a,
\end{aligned}
$$

with

$$
d_{\text {n.a. }}(a)=a \tan \theta\left(\frac{1-\sin \varphi_{0}}{\cos \varphi_{0}}+\varphi_{0}\right) .
$$

The relationships (3.105) to (3.108) are all valid only for $a \geq b$. If $a<b$, the contact resembles the one with a paraboloid, for which results can be looked up in 
Sect. 3.5.3. In the previous chapter, this distinction was ignored because of its triviality. For the adhesive contact, however, it does not have quite trivial consequences for the stability of the contact. The non-adhesive indentation depth is completely described by:

$$
d_{\text {n.a. }}(a)= \begin{cases}\frac{a^{2} \tan \theta}{b}, & a \leq b, \\ a \tan \theta\left(\frac{1-\sin \varphi_{0}}{\cos \varphi_{0}}+\varphi_{0}\right), & a>b .\end{cases}
$$

According to (3.23), the contact radius at which the contact loses its stability results as a solution of the equation:

$$
2 \frac{1-\sin \varphi_{0 . c}}{\cos \varphi_{0, c}}+\varphi_{0, c}-\beta \sqrt{\cos \varphi_{0, c}}=0,
$$

with

$$
\beta:=\frac{\xi}{\tan \theta} \sqrt{\frac{\pi \Delta \gamma}{2 E^{*} b}},
$$

if that equation has solutions. The corresponding indentation depth is given by:

$$
d_{c}=a \tan \theta\left[\frac{1-\sin \varphi_{0, c}}{\cos \varphi_{0, c}}\left(1-\frac{4}{\xi}\right)+\varphi_{0, c}\left(1-\frac{2}{\xi}\right)\right],
$$

and the corresponding normal force by:

$$
\begin{aligned}
F_{c}=E^{*} a^{2} \tan \theta & {\left[\varphi_{0, c}\left(1-\frac{4}{\xi}\right)+\frac{1-\sin \varphi_{0, c}}{\cos \varphi_{0}, c}\left(\frac{4}{3}-\frac{8}{\xi}\right)\right.} \\
& \left.+\frac{1}{3} \sin \varphi_{0, c} \cos \varphi_{0, c}\right] .
\end{aligned}
$$

As expected, the solutions of the cone and the paraboloid from Sects. 3.5.2 and 3.5.3 are obtained by setting $b=0$ or $b=a$ correspondingly (in this case it is $R:=b / \tan \theta) . \quad \xi$ is a parameter which determines the type of boundary condition (force or displacement-control; see (3.23)).

It turns out that, when solving (3.110), there are three different regimes: For $\beta<1.795$ there is no solution and the critical contact radius is smaller than $b$. This means that the relationships for the critical state match those of the parabolic indenter that can be looked up in Sect. 3.5.3. If $1.795 \leq \beta \leq 2$, there are two solutions for the equation which are both shown in Fig. 3.20 and, correspondingly, two critical states. For values $\beta>2$ only the larger of the two solutions remains. The smaller one becomes negative and thus unphysical. In this case, the curves of the normalized critical values of contact radius, indentation depth, and normal force in the case of force-control are given in Fig. 3.21. For formatting reasons, a sign change was made during the normalization of the "adhesion force" $F_{c}$. 
Fig. 3.20 Stability bifurcation of the angle $\varphi_{0}$ as a function of the normalized surface energy $\beta$ (see (3.111)) for the adhesive indentation by a cone with a rounded tip

Fig. 3.21 Curves of the normalized critical values for the contact radius $a_{c} / b$, the indentation depth $d_{c} /(b \tan \theta)$, and the normal force $-F_{c} /\left(4 E^{*} b^{2} \tan \theta\right)$ as a function of $\beta$ for the indentation by a cone with a rounded tip during forcecontrol. Note the negative sign of the normalized force
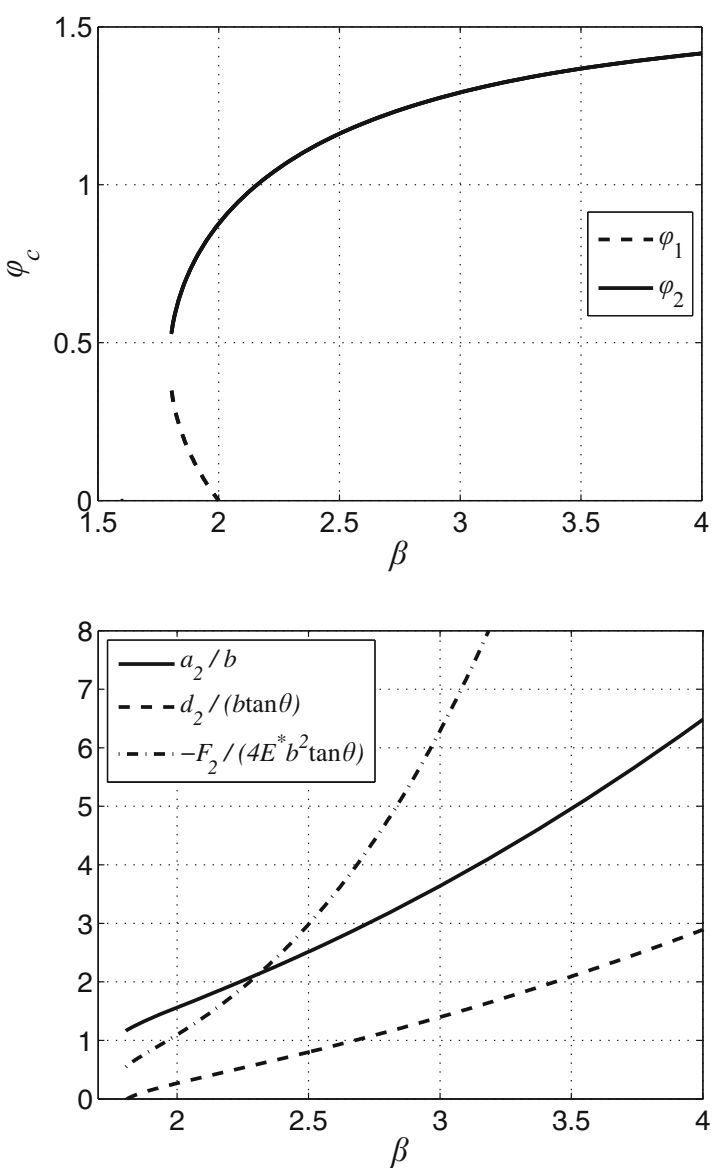

\subsubsection{The Paraboloid with Parabolic Cap}

Also, for the paraboloid with a spherical cap whose radius is greater than the radius of curvature of the parabolic base body, Chap. 2 presented the complete solution of the non-adhesive, frictionless, normal contact problem (see Sect. 2.5.13). Thus, from (3.18) and (3.20), the following solution which was first found by Maugis and Barquins (1983) results in the adhesive, frictionless, normal contact:

$$
\begin{aligned}
d(a) & =\frac{a^{2}}{R_{1}}+\frac{a}{R^{*}} \sqrt{a^{2}-b^{2}}-\sqrt{\frac{2 \pi a \Delta \gamma}{E^{*}}}, \\
F_{N}(a) & =\frac{2 E^{*}}{3}\left[\frac{2 a^{3}}{R_{1}}+\frac{1}{R^{*}}\left(2 a^{2}+b^{2}\right) \sqrt{a^{2}-b^{2}}\right]-\sqrt{8 \pi a^{3} E^{*} \Delta \gamma},
\end{aligned}
$$




$$
\begin{gathered}
\sigma_{z z}(r ; a)=\sigma_{z z \text {,n.a. }}(r ; a)+\sqrt{\frac{2 E^{*} \Delta \gamma}{\pi a}} \frac{a}{\sqrt{a^{2}-r^{2}}}, \quad r \leq a, \\
w(r ; a)=w_{\text {n.a. }}(r ; a)-\sqrt{\frac{8 a \Delta \gamma}{\pi E^{*}}} \arcsin \left(\frac{a}{r}\right), \quad r>a .
\end{gathered}
$$

Here, $b$ denotes the radius at which the cap goes over into the base body; $R^{*}$ is an effective radius that can be determined from $R_{1}$ and $R_{2}$ (see Fig. 3.22):

$$
R^{*}:=\frac{R_{1} R_{2}}{R_{1}-R_{2}} .
$$

The stresses and displacements indicated by the index "n.a." correspond to the solutions of the non-adhesive problem (see Sect. 2.5.13):

$$
\begin{gathered}
\sigma_{z z \text {,n.a. }}(r ; a)=-\frac{E^{*}}{\pi}\left\{\begin{array}{l}
\frac{2 \sqrt{a^{2}-r^{2}}}{R_{1}}+\int_{b}^{a} \frac{\left(2 x^{2}-b^{2}\right) \mathrm{d} x}{R^{*} \sqrt{x^{2}-b^{2}} \sqrt{x^{2}-r^{2}}}, \quad r \leq b, \\
\frac{2 \sqrt{a^{2}-r^{2}}}{R_{1}}+\int_{r}^{a} \frac{\left(2 x^{2}-b^{2}\right) \mathrm{d} x}{R^{*} \sqrt{x^{2}-b^{2}} \sqrt{x^{2}-r^{2}}}, \quad b<r \leq a,
\end{array}\right. \\
w_{\text {n.a. }}(r ; a)=w_{\text {n.a., } P}\left(r ; a ; R=R_{1}\right)+w_{\text {n.a., } P S}\left(r ; a ; R=R^{*}\right), \quad r>a, \quad
\end{gathered}
$$

with the corresponding solutions for the paraboloid and the truncated paraboloid:

$$
\begin{aligned}
& w_{\text {n.a. }, P}\left(r ; a ; R_{1}\right):= \frac{a^{2}}{\pi R_{1}}\left[\left(2-\frac{r^{2}}{a^{2}}\right) \arcsin \left(\frac{a}{r}\right)+\frac{\sqrt{r^{2}-a^{2}}}{a}\right], \\
& w_{\text {n.a., } P S}\left(r ; a ; R^{*}\right):= \frac{2 a}{\pi R^{*}} \sqrt{a^{2}-b^{2}} \arcsin \left(\frac{a}{r}\right) \\
&-\frac{1}{\pi R^{*}}\left[\left(r^{2}-b^{2}\right) \arcsin \left(\frac{\sqrt{a^{2}-b^{2}}}{\sqrt{r^{2}-b^{2}}}\right)\right. \\
&\left.-\sqrt{a^{2}-b^{2}} \sqrt{r^{2}-a^{2}}\right] .
\end{aligned}
$$

Fig. 3.22 Adhesive normal contact between a rigid paraboloid with parabolic cap and an elastic half-space

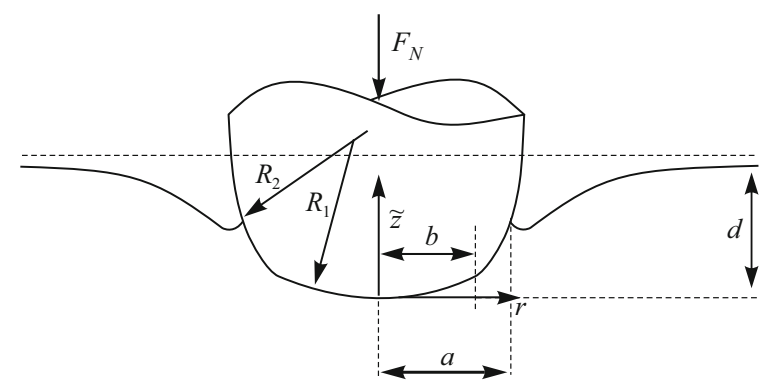


All relations in (3.114) to (3.117) are valid only for $a \geq b$. If $a<b$ contact is made only with a parabolic indenter of radius $R_{1}$. The indentation depth in the case without adhesion is thus completely given by:

$$
d_{\text {n.a. }}(a)=\left\{\begin{array}{ll}
\frac{a^{2}}{R_{1}}, & a \leq b, \\
\frac{a^{2}}{R_{1}}+\frac{a}{R^{*}} \sqrt{a^{2}-b^{2}}, & a>b,
\end{array}= \begin{cases}\frac{a^{2}}{R_{1}}, & a \leq b, \\
\frac{a^{2}}{R_{1}}\left(1+\frac{R_{1}}{R^{*}} \sin \varphi_{0}\right), & a>b,\end{cases}\right.
$$

where we introduced the angle:

$$
\varphi_{0}:=\arccos \left(\frac{b}{a}\right) .
$$

The critical configuration in which the adhesive normal contact loses its stability results from the solution to the transcendental equation:

$$
\frac{1}{\cos \varphi_{0, c}}\left(2+\frac{R_{1}}{R^{*}} \frac{1+\sin ^{2} \varphi_{0, c}}{\sin \varphi_{0, c}}\right)-\beta \sqrt{\cos \varphi_{0, c}}=0,
$$

with

$$
\beta:=\frac{\xi R_{1}}{b} \sqrt{\frac{\pi \Delta \gamma}{2 E^{*} b}} .
$$

The corresponding values of the indentation depth and the normal force are determined by:

$$
\begin{aligned}
& d_{c}=\frac{a_{c}^{2}}{R_{1}}\left[1-\frac{4}{\xi}+\frac{R_{1}}{R^{*}} \sin \varphi_{0, c}\left(1-\frac{2}{\xi}-\frac{2}{\xi \sin ^{2} \varphi_{0, c}}\right)\right], \\
& F_{c}=\frac{4 E^{*} a_{c}^{3}}{3 R_{1}}\left\{1-\frac{6}{\xi}+\frac{R_{1}}{2 R^{*}} \sin \varphi_{0, c}\right. {\left[3-\sin ^{2} \varphi_{0, c}\right.} \\
&\left.\left.-\frac{6}{\xi}\left(1+\frac{1}{\sin ^{2} \varphi_{0, c}}\right)\right]\right\},
\end{aligned}
$$

and one easily convinces oneself that the following limiting cases emerge from these solutions:

- For $R_{1} \rightarrow \infty$, the truncated paraboloid from Sect. 3.5.9

- For $R^{*} \rightarrow \infty$, the paraboloid with curvature radius $R_{1}$ from Sect. 3.5.3

- For $b=0$ the paraboloid with radius of curvature $R_{2}$

In (3.120) and (3.122), $\xi$ (i.e., the boundary condition) has to be defined: displacement-controlled trials corresponds to the value $\xi=1$, and force-controlled ones to $\xi=3$. 
Fig. 3.23 First solution for the critical angle $\varphi_{0, c}$, in which the contact becomes unstable, depending on the parameters $R_{1} / R_{1}$ and $\beta$ (see (3.121)), for adhesive indentation by a paraboloid with parabolic cap

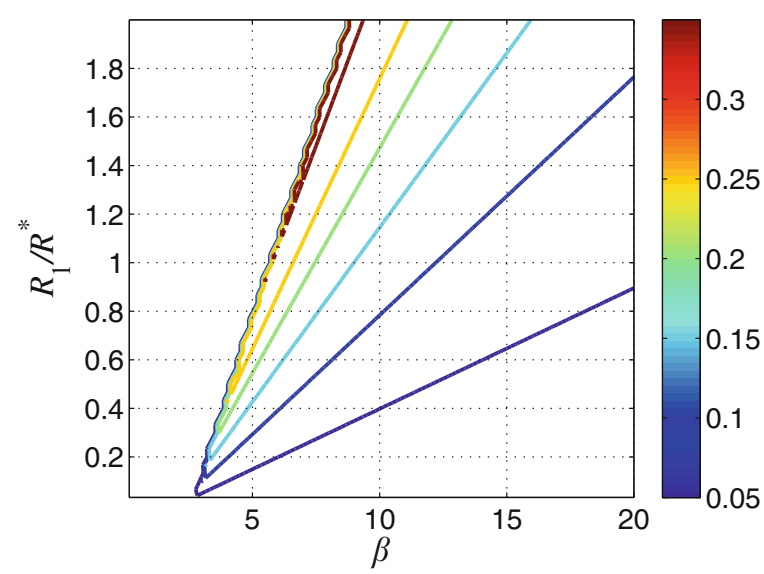

Fig. 3.24 Second solution for the critical angle $\varphi_{0, c}$ in which the contact becomes unstable, depending on the parameters $R_{1} / R_{1}$ and $\beta$, for adhesive indentation by a paraboloid with parabolic cap

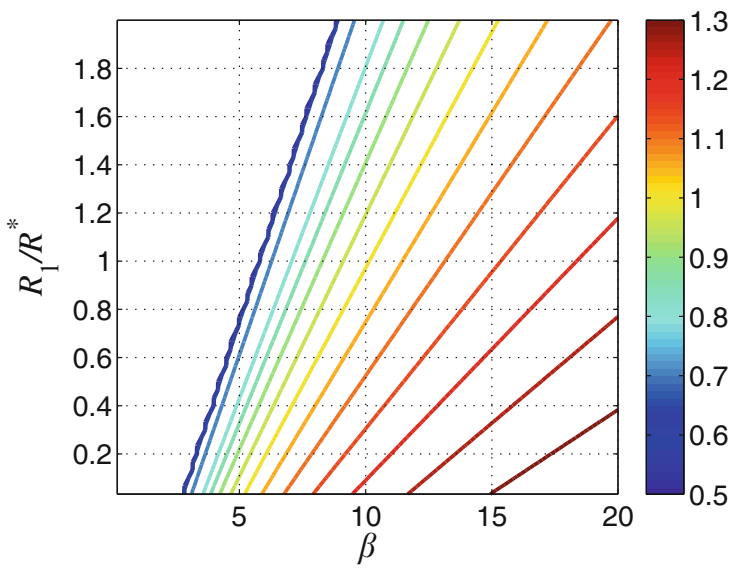

In (3.120), two dimensionless parameters occur; $R_{1} / R^{*}$ and $\beta$. The equation has, with one exception, either no solution or two solutions. These two solutions are shown in Figs. 3.23 and 3.24. It can be seen that there are only solutions below the line:

$$
\beta>\beta_{0}\left(\frac{R_{1}}{R^{*}}\right) \approx 2.2+3.2 \frac{R_{1}}{R^{*}} .
$$

For smaller values of the surface energy, and thus smaller values of $\beta$, the critical contact radius is smaller than $b$ and the critical state corresponds to that of the complete paraboloid with the radius of curvature $R_{1}$, for which the results can be found in Sect. 3.5.3. From Sect. 3.5.9 it can be deduced that:

$$
\beta_{0}\left(\frac{R_{1}}{R^{*}} \rightarrow \infty\right) \approx 3.1 \frac{R_{1}}{R^{*}}
$$


This agrees relatively well with the estimate (3.123). For $R_{1} / R^{*}=0$, the first solution $\varphi_{0, c 1}$ becomes an unphysical artifact, leaving the one critical state for the indentation by a purely parabolic indenter.

\subsubsection{The Cylindrical Flat Punch with a Rounded Edge}

For the flat cylindrical punch with a round edge, the solution of the non-adhesive Boussinesq problem was derived in Chap. 2 (Sect. 2.5.14). From (3.18) and (3.20), we obtain the following solution for the adhesive problem (see Fig. 3.25):

$$
\begin{aligned}
d(a)= & \frac{a}{R}\left[\sqrt{a^{2}-b^{2}}-b \arccos \left(\frac{b}{a}\right)\right]-\sqrt{\frac{2 \pi a \Delta \gamma}{E^{*}}}, \\
F_{N}(a)= & \frac{E^{*}}{3 R}\left[\sqrt{a^{2}-b^{2}}\left(4 a^{2}-b^{2}\right)-3 b a^{2} \arccos \left(\frac{b}{a}\right)\right] \\
& -\sqrt{8 \pi a^{3} E^{*} \Delta \gamma}, \\
\sigma_{z z}(r ; a)= & \sigma_{z z \text {,n.a. }}(r ; a)+\sqrt{\frac{2 E^{*} \Delta \gamma}{\pi a}} \frac{a}{\sqrt{a^{2}-r^{2}}}, \quad r \leq a, \\
w(r ; a)= & w_{\text {n.a. }}(r ; a)-\sqrt{\frac{8 a \Delta \gamma}{\pi E^{*}}} \arcsin \left(\frac{a}{r}\right), \quad r>a .
\end{aligned}
$$

Here, $b$ denotes the radius of the flat base of the punch and $R$ the radius of curvature of the rounded corners. The stresses and displacements are indicated by the index

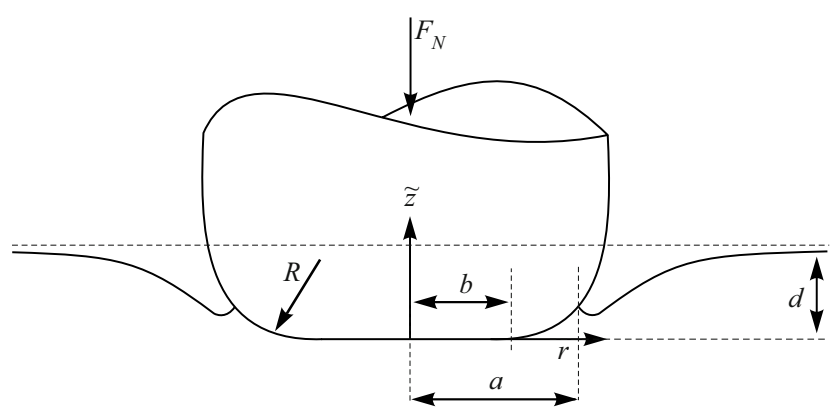

Fig. 3.25 Adhesive normal contact between a rigid cylindrical punch with round corners and an elastic half-space 
"n.a." for the problem without adhesion are (see Sect. 2.5.14):

$$
\begin{aligned}
& \sigma_{z z \text {,n.a. }}(r ; a)= \\
& -\frac{E^{*}}{\pi R}\left\{\begin{array}{l}
\int_{b}^{a}\left(2 \sqrt{x^{2}-b^{2}}-b \arccos \left(\frac{b}{x}\right)\right) \frac{\mathrm{d} x}{\sqrt{x^{2}-r^{2}}}, \quad r \leq b, \\
\int_{r}^{a}\left(2 \sqrt{x^{2}-b^{2}}-b \arccos \left(\frac{b}{x}\right)\right) \frac{\mathrm{d} x}{\sqrt{x^{2}-r^{2}}}, \quad b<r \leq a,
\end{array}\right. \\
& \begin{array}{l}
w_{\text {n.a. }}(r ; a)= \\
\frac{2 d_{\text {n.a. }}(a)}{\pi} \arcsin \left(\frac{a}{r}\right)-\frac{2}{\pi}\left[\int_{b}^{a} \frac{x}{R}\left(\sqrt{x^{2}-b^{2}}-b \arccos \left(\frac{b}{x}\right)\right) \frac{\mathrm{d} x}{\sqrt{r^{2}-x^{2}}}\right], \\
r>a,
\end{array}
\end{aligned}
$$

with the non-adhesive relationship between indentation depth and contact radius being:

$$
d_{\text {n.a. }}(a)=\frac{a}{R}\left\{\sqrt{a^{2}-b^{2}}-b \arccos \left(\frac{b}{a}\right)\right\}=\frac{a^{2}}{R}\left(\sin \varphi_{0}-\varphi_{0} \cos \varphi_{0}\right),
$$

with the angle:

$$
\varphi_{0}:=\arccos \left(\frac{b}{a}\right) .
$$

In contrast to Sects. 3.5.11 and 3.5.12, here $a$ can never be smaller than $b$. The critical contact radius is given as a solution of:

$$
2 \tan \varphi_{0, c}-\varphi_{0, c}-\beta \sqrt{\cos \varphi_{0, c}}=0,
$$

with:

$$
\beta:=\frac{\xi R}{b} \sqrt{\frac{\pi \Delta \gamma}{2 E^{*} b}} .
$$

The corresponding values of the indentation depth and the normal force are determined by:

$$
\begin{gathered}
d_{c}=\frac{a_{c}^{2}}{R}\left[\sin \varphi_{0, c}\left(1-\frac{4}{\xi}\right)-\varphi_{0, c} \cos \varphi_{0, c}\left(1-\frac{2}{\xi}\right)\right], \\
F_{c}=\frac{4 E^{*} a_{c}^{3}}{3 R}\left[\sin \varphi_{0, c}\left(1-\frac{6}{\xi}-\frac{1}{4} \cos ^{2} \varphi_{0, c}\right)\right. \\
\left.-\frac{3}{4} \varphi_{0, c} \cos \varphi_{0, c}\left(1-\frac{4}{\xi}\right)\right] .
\end{gathered}
$$

For $b=0$ the solution for the parabolic indenter from Sect. 3.5.3 is reproduced. $\xi$ is a parameter that, depending on the nature of the boundary condition, can take the values one (displacement-control) and three (force-control). 
Fig. 3.26 Normalized critical values of the angle $\varphi_{0}$ the contact radius, the indentation depth, and normal force as a function of the parameter $\beta$ (see (3.130)) for adhesive indentation by a cylindrical flat punch with rounded corners

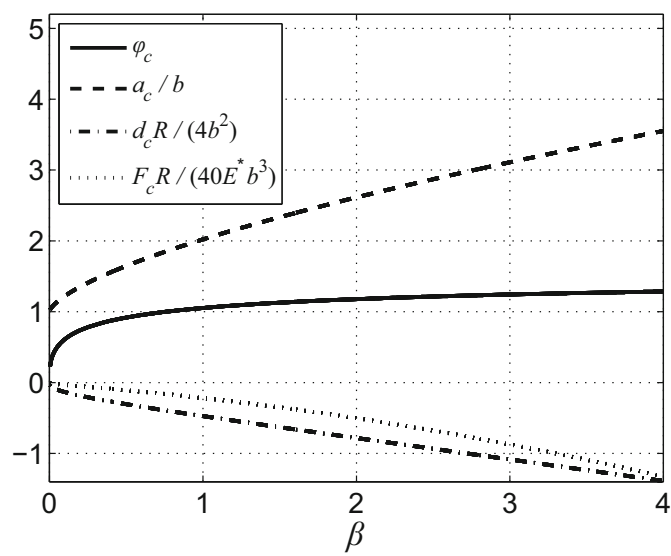

Interestingly, (3.129) has only one solution for each positive $\beta$. This and the associated normalized values of $a_{c}, d_{c}$, and $F_{c}$ are shown in Fig. 3.26. For $\beta \rightarrow 0$ the critical values of the indentation depth and the "adhesion force" disappear, whereas the critical contact radius does not disappear but strives towards its smallest possible value, $b$. This is due to the fact that the rounded corners, which are continuously differentiable (in contrast to the truncated paraboloid), pass into the flat base parallel to the half-space.

\subsubsection{The Paraboloid with Small Periodic Roughness (Complete Contact)}

It is established that the adhesive properties of a surface are greatly affected by the surface roughness. In general, increasing roughness sees a reduction in the effective surface energy and, therefore, the adhesive forces. However, there exists much proof in existing literature, such as that by Briggs and Briscoe (1977) demonstrating that the opposite can also be the case. A theoretical yet experimentally validated approach explaining this phenomenon stems from Guduru (2007). With the aid of both the classical approach of minimizing the potential energy by Johnson et al. (1971) as well as the idea of elastic energy release rates borrowed from linear-elastic fracture mechanics, he examined the adhesive normal indentation of a parabolic indenter with periodic roughness. It can be represented by the profile:

$$
f(r)=\frac{r^{2}}{2 R}+h\left(1-\cos \left(\frac{2 \pi}{\lambda} r\right)\right),
$$

with the amplitude $h$ and wavelength $\lambda$ of the roughness, and the radius $R$ of the paraboloid. For his solution, Guduru assumed a simply connected contact area requiring a monotonically increasing indenter profile. This poses a limitation for 
the roughness parameters:

$$
f^{\prime}(r) \geq 0 \Rightarrow \frac{1}{\hat{h}}:=\frac{\lambda^{2}}{h R} \geq 4 \pi^{2} \sup \left[-\frac{\sin (x)}{x}\right] \approx 8.576 .
$$

Here, sup [·] denotes the global maximum of a function. However, a sufficiently great normal force can overcome this to generate a connected contact area, even for profiles that violate this condition. Taking into account the solution of the nonadhesive contact problem from Chap. 2 (Sect. 2.5.17) and (3.18) and (3.20), the solution for the adhesive problem is then as follows:

$$
\begin{aligned}
d(a)= & \frac{a^{2}}{R}+\frac{\pi^{2} h a}{\lambda} \mathrm{H}_{0}\left(\frac{2 \pi}{\lambda} a\right)-\sqrt{\frac{2 \pi a \Delta \gamma}{E^{*}}}, \\
F_{N}(a)= & \frac{4 E^{*} a^{3}}{3 R}+E^{*} \pi a h\left[\frac{2 \pi}{\lambda} a \mathrm{H}_{0}\left(\frac{2 \pi}{\lambda} a\right)-\mathrm{H}_{1}\left(\frac{2 \pi}{\lambda} a\right)\right] \\
& -\sqrt{8 \pi a^{3} E^{*} \Delta \gamma}, \\
\sigma_{z z}(r ; a)= & \sigma_{z z, \text { n.a. }}(r, a)+\sqrt{\frac{2 E^{*} \Delta \gamma}{\pi a}} \frac{a}{\sqrt{a^{2}-r^{2}}}, \quad r \leq a, \\
w(r ; a)= & w_{\text {n.a. }}(r, a)-\sqrt{\frac{8 a \Delta \gamma}{\pi E^{*}}} \arcsin \left(\frac{a}{r}\right), \quad r>a .
\end{aligned}
$$

Here, $H_{n}(\cdot)$ denotes the $n$-th order Struve function. For a short explanation of its properties, the reader can be referred to the solution of the non-adhesive problem detailed in Sect. 2.5.17. The stresses and displacements for the non-adhesive case can also be referenced in Sect. 2.5.17 and are repeated below:

$$
\begin{aligned}
& \sigma_{z z \text {,n.a. }}(r ; a)= \\
& -\frac{E^{*}}{\pi}\left\{\frac{2 \sqrt{a^{2}-r^{2}}}{R}+\frac{\pi^{2} h}{\lambda} \int_{r}^{a}\left[\mathrm{H}_{0}\left(\frac{2 \pi}{\lambda} x\right)+\frac{2 \pi}{\lambda} x \mathrm{H}_{-1}\left(\frac{2 \pi}{\lambda} x\right)\right] \frac{\mathrm{d} x}{\sqrt{x^{2}-r^{2}}}\right\}, \\
& \quad r \leq a, \\
& w_{\text {n.a. }}(r ; a)=\frac{2 d_{\text {n.a. }}(a)}{\pi} \arcsin \left(\frac{a}{r}\right)-\frac{1}{\pi R}\left[r^{2} \arcsin \left(\frac{a}{r}\right)-a \sqrt{r^{2}-a^{2}}\right] \\
& \quad-\frac{2 \pi}{\lambda} h \int_{0}^{a} x \mathrm{H}_{0}\left(\frac{2 \pi}{\lambda} x\right) \frac{\mathrm{d} x}{\sqrt{r^{2}-x^{2}}}, \quad r>a,
\end{aligned}
$$

with

$$
d_{\text {n.a. }}(a)=\frac{a^{2}}{R}+\frac{\pi^{2} h a}{\lambda} \mathrm{H}_{0}\left(\frac{2 \pi}{\lambda} a\right) .
$$


Fig. 3.27 Relationship between the normalized normal force and the normalized indentation depth for the adhesive contact with a paraboloid with periodic roughness for different normalized values of roughness

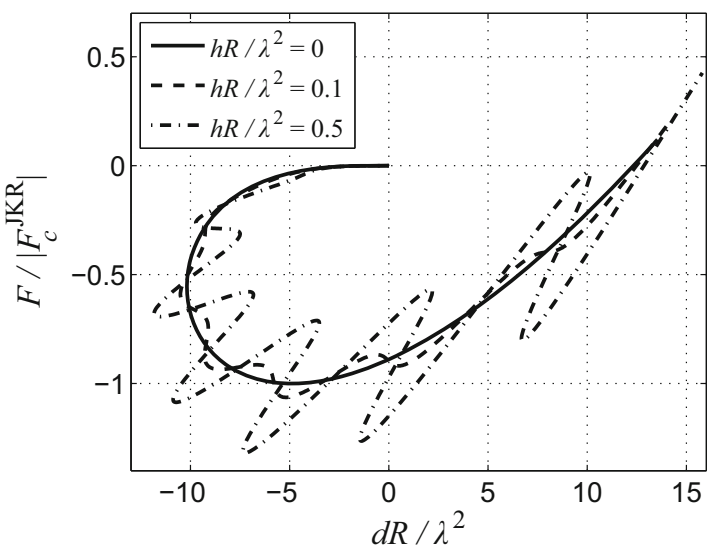

By introducing the dimensionless quantities

$$
\hat{d}:=\frac{d R}{\lambda^{2}}, \quad \hat{a}:=\frac{a}{\lambda}, \quad \hat{h}:=\frac{h R}{\lambda^{2}}, \quad \hat{F}:=\frac{2 F_{N}}{3 \pi R \Delta \gamma}, \quad \overline{\Delta \gamma}:=\frac{2 \pi \Delta \gamma R^{2}}{E^{*} \lambda^{3}},
$$

the relationships $\left(3.134_{1}\right)$, and $\left(3.134_{2}\right)$, can be rewritten as:

$$
\begin{aligned}
& \hat{d}=\hat{a}^{2}+\pi^{2} \hat{h} \hat{a} \mathrm{H}_{0}(2 \pi \hat{a})-\sqrt{\overline{\Delta \gamma} \hat{a}}, \\
& \hat{F}=\frac{16}{9 \overline{\Delta \gamma}} \hat{a}^{3}+\frac{4 \pi}{3} \frac{\hat{h} \hat{a}}{\overline{\Delta \gamma}}\left[2 \pi \hat{a} \mathrm{H}_{0}(2 \pi \hat{a})-\mathrm{H}_{1}(2 \pi \hat{a})\right]-\frac{8}{3} \sqrt{\frac{\hat{a}^{3}}{\overline{\Delta \gamma}}} .
\end{aligned}
$$

The implicitly defined relationship $\hat{F}=\hat{F}(\hat{d})$ is partially graphically represented in Fig. 3.27.

The wavelength is kept constant for the three depicted cases: the JKR case without roughness $h=0$, one curve with medium roughness, and one with very pronounced roughness. We discover that the force oscillates with increasing magnitude. On the one hand, we see significantly greater extremes for the adhesive forces (during force-control) and indentation depth (during displacement-control) being achieved than without roughness (lowest point of the curve with $\hat{h}=0.5$ lies at $\hat{F}=-1.3$, roughly $30 \%$ lower than the minimum of the JKR curve). On the other hand, constantly switching between the stable and unstable regime causes the indenter to bounce, which is a process that dissipates energy. 


\subsection{Adhesion According to Bradley}

Let us consider a rigid paraboloid $\tilde{z}=r^{2} /(2 R)$ in contact with a rigid plane. Further, we assume that the adhesive stress only depends on the distance between the surface: $\sigma=\sigma(s)$. The separation energy is then:

$$
\Delta \gamma=\int_{0}^{\infty} \sigma(s) \mathrm{d} s
$$

For the adhesive force between the spheres we obtain:

$$
F_{A}=\int_{0}^{\infty} 2 \pi r \sigma[s(r)] \mathrm{d} r=\int_{0}^{\infty} 2 \pi r \sigma\left(\frac{r^{2}}{2 R}\right) \mathrm{d} r .
$$

By substituting $s=r^{2} /(2 R), r \mathrm{~d} r=R \mathrm{~d} s$ the equation is transformed into the following form:

$$
F_{A}=2 \pi R \int_{0}^{\infty} \sigma(s) \mathrm{d} s=2 \pi R \Delta \gamma .
$$

This result was derived by Bradley (1932), specifically for the van der Waals interaction, while in reality it is independent of the type of interaction potential.

\subsection{Adhesion According to Derjaguin, Muller, and Toporov}

Derjaguin, Muller, and Toporov (1975) examined the adhesive normal contact between a paraboloid of radius $R$ and an elastic half-space. They assumed that the stresses within the contact area and the deformation outside the contact area matched those found in the non-adhesive Hertzian solution. Of course, this implied that the body forms an ideal sphere in the last moment of the contact. As a result, the pull-off force in this state is identical to the force calculated by Bradley:

$$
F_{A}=2 \pi R \Delta \gamma
$$

\subsection{Adhesion According to Maugis}

As explained in the introduction to this chapter, the adhesive forces between two electrically neutral bodies decrease rapidly with increasing distance. Therefore, integrating with respect to distance (i.e., the work of adhesion) returns a definite value. Adhesive forces that are of much shorter range than any other characteristic length of the problem have a negligible impact and are assumed to be zero. In this case-corresponding to the JKR approximation-the work of adhesion is 
the sole determinant of the adhesive behavior. The adhesive forces attain distinct significance once the range of the adhesive forces is of the order of the smallest characteristic length of the contact problem. The adhesive behavior is now determined not only by the work of adhesion but also independently by both the intensity and range of the adhesive forces. In examining these influences in a qualitative fashion, it is sensible to begin with the easiest model of finitely ranged adhesive forces: by assuming that the adhesive stresses (adhesive force divided by area) are constant up to a certain distance and zero past that point. This approximation was introduced by Dugdale (1960) to analyze crack problems and has since seen heavy usage due to its simplicity. The theory of adhesion based on the aforementioned adhesive force was developed by Maugis (1992). In the following section, the theory of Maugis will be derived via the MDR.

\subsubsection{General Solution for the Adhesive Contact of Axisymmetric Bodies in Dugdale Approximation}

We will consider the adhesive contact between a rigid rotationally symmetric profile with the shape $f(r)$ and an elastic half-space. The solution is found via MDR and (except for the interpretation of the MDR model) it is identical to the original solution by Maugis. Executing the MDR algorithm, we define an effective profile $g(x)($ see $(2.6))$ :

$$
g(x)=|x| \int_{0}^{|x|} \frac{f^{\prime}(r)}{\sqrt{x^{2}-r^{2}}} \mathrm{~d} r .
$$

This will play a central role in the following solution steps. This profile is brought into contact with a one-dimensional Winkler foundation, defined by (2.5). In contrast to the non-adhesive contact, there now exists effective adhesive forces acting between the indenter and the Winkler foundation.

While pressure is the relevant quantity of load in three-dimensional space, the one-dimensional MDR representation only allows the definition of the linear force density (distributed load):

$$
q_{z}(x)=\frac{\Delta F_{N}(x)}{\Delta x}=E^{*} w_{1 D}(x) .
$$

Between these two quantities, the following transformations are valid:

$$
\begin{aligned}
& p(r)=-\frac{1}{\pi} \int_{r}^{\infty} \frac{q_{z}^{\prime}(x)}{\sqrt{x^{2}-r^{2}}} \mathrm{~d} x, \\
& q_{z}(x)=2 \int_{x}^{\infty} \frac{r p(r)}{\sqrt{r^{2}-x^{2}}} \mathrm{~d} r .
\end{aligned}
$$


These are described in detail in Chap. 2 (Sect. 2.3) and in the appendix (Chap. 11). The displacements of the surface points in the original three-dimensional problem $w(r)$ and in the one-dimensional MDR representation $w_{1 D}(x)$ are linked via the usual MDR transformation:

$$
w(r)=\frac{2}{\pi} \int_{0}^{r} \frac{w_{1 D}(x)}{\sqrt{r^{2}-x^{2}}} \mathrm{~d} x .
$$

Following Maugis's (1992) lead, we will examine the case of a constant adhesive stress with finite range, i.e., we assume that the adhesive pressure in the original three-dimensional system remains constant and equal to the magnitude of $\sigma_{0}$ up to the distance $h$, and vanishing thereafter:

$$
p_{\text {adh }}(r)= \begin{cases}-\sigma_{0}, & \text { for } f(r)-d+w(r) \leq h, \\ 0, & \text { for } f(r)-d+w(r)>h .\end{cases}
$$

Using the transformations (3.145) we can determine the corresponding "adhesive distributed load" in the one-dimensional MDR model:

$$
q_{\mathrm{adh}, z}(x)= \begin{cases}-2 \int_{x}^{b} \frac{r \sigma_{0}}{\sqrt{r^{2}-x^{2}}} \mathrm{~d} r=-2 \sigma_{0} \sqrt{b^{2}-x^{2}}, & \text { for }|x| \leq b, \\ 0, & \text { for }|x|>b,\end{cases}
$$

where $b$ denotes the outer radius of the area upon which the adhesive pressure acts (i.e., where the distance between the surfaces does not exceed $h$ ). The radius $b$ depends on the deformation of the surfaces and is calculated as part of the solution of the contact problem. The geometry of the contact and the notation is graphically represented in Fig. 3.28.

Within the contact area, the displacement $w_{1 D}(x)$ of the $1 \mathrm{D}$ model is determined by the shape of the indenter and outside the contact area (but still within the adhesive zone) by the adhesive forces:

$$
w_{1 D}(x)= \begin{cases}d-g(x), & x \leq a, \\ -\frac{2 \sigma_{0}}{E^{*}} \sqrt{b^{2}-x^{2}}, & a<x \leq b, \\ 0, & x>b .\end{cases}
$$

The radius of the adhesive zone derives from the condition that the gap $w(b)-d+f(b)$ between the indenter and the elastic body must correspond to the range $h$ of the adhesive stresses:

$$
w(b)-d+f(b)=h .
$$



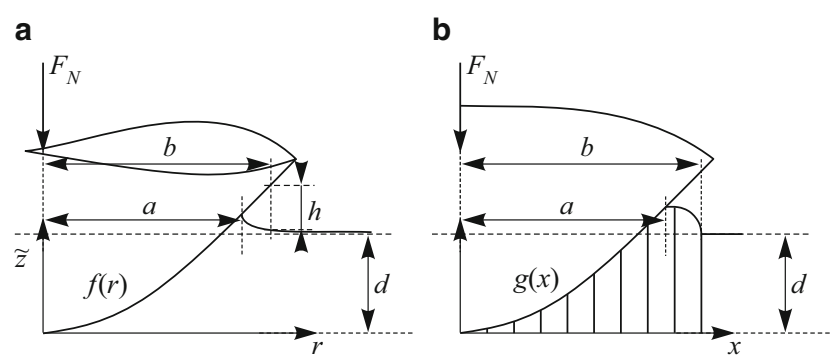

Fig. 3.28 Adhesive contact according to the Dugdale-Maugis model: subfigure (a) represents the real contact with the three-dimensional half-space, and subfigure (b) the contact with the one-dimensional Winkler foundation. In three dimensions the constant attractive stress $\sigma_{0}$ acts wherever the distance between the surfaces is less than $h$. At greater distances the interactions cease. The radius of the adhesive zone exceeds the contact radius $a$. In the MDR representation the "adhesive distributed load" $q_{\text {adh }}(x)$ (3.148) takes the place of the adhesive stress

Accounting for the transformation (3.146) and (2.149), we get the displacement $w(b)$ (3.149):

$$
\begin{aligned}
w(b) & =\frac{2}{\pi} \int_{0}^{b} \frac{w_{1 D}(x)}{\sqrt{b^{2}-x^{2}}} \mathrm{~d} x \\
& =d-f(b)-\frac{2}{\pi} \int_{a}^{b} \frac{[d-g(x)]}{\sqrt{b^{2}-x^{2}}} \mathrm{~d} x-\frac{4 \sigma_{0}}{\pi E^{*}}(b-a) .
\end{aligned}
$$

Inserting this into (3.150) then yields the equation for the radius $b$ :

$$
\frac{2}{\pi} \int_{a}^{b} \frac{[g(x)-d]}{\sqrt{b^{2}-x^{2}}} \mathrm{~d} x-\frac{4 \sigma_{0}}{\pi E^{*}}(b-a)=h .
$$

The force $\Delta F_{\text {cont }, z}(x)$ pressing the spring at coordinate $x$ onto the effective profile $g(x)$ consists of the elastic spring force and the adhesive force:

$$
\begin{aligned}
\Delta F_{\mathrm{cont}, z}(x) & =\Delta x\left[q_{z}(x)-q_{\mathrm{adh}, z}(x)\right] \\
& =\Delta x\left\{E^{*}[d-g(x)]-q_{\mathrm{adh}, z}(x)\right\} .
\end{aligned}
$$

The contact radius $a$ is obtained from the condition that this contact force disappears:

$$
E^{*}[d-g(a)]+2 \sigma_{0} \sqrt{b^{2}-a^{2}}=0 .
$$

Furthermore, the total normal force is calculated as the integral over all springs:

$$
F_{N}=2 E^{*} \int_{0}^{b} w_{1 D}(x) \mathrm{d} x .
$$


Substituting (3.149) gives:

$$
\begin{aligned}
F_{N} & =2 E^{*} \int_{0}^{b} w_{1 D}(x) \mathrm{d} x=2 E^{*} \int_{0}^{a}[d-g(x)] \mathrm{d} x-4 \sigma_{0} \int_{a}^{b} \sqrt{b^{2}-x^{2}} \mathrm{~d} x \\
& =2 E^{*} \int_{0}^{a}[d-g(x)] \mathrm{d} x-\sigma_{0}\left[-2 a \sqrt{b^{2}-a^{2}}+2 b^{2} \arccos \left(\frac{a}{b}\right)\right] .
\end{aligned}
$$

Equations (3.152), (3.154), and (3.156) completely solve the normal contact problem. Here, we will summarize them once more:

$$
\begin{aligned}
h= & \frac{2}{\pi} \int_{a}^{b} \frac{[g(x)-d]}{\sqrt{b^{2}-x^{2}}} \mathrm{~d} x-\frac{4 \sigma_{0}}{\pi E^{*}}(b-a), \\
0= & E^{*}[d-g(a)]+2 \sigma_{0} \sqrt{b^{2}-a^{2}}, \\
F_{N}= & 2 E^{*} \int_{0}^{a}[d-g(x)] \mathrm{d} x \\
& -\sigma_{0}\left[-2 a \sqrt{b^{2}-a^{2}}+2 b^{2} \arccos \left(\frac{a}{b}\right)\right] .
\end{aligned}
$$

\subsubsection{The JKR Limiting Case for Arbitrary Axisymmetric Indenter Shapes}

In this case $\sigma_{0} \rightarrow \infty$ and $h \rightarrow 0$, where $\sigma_{0} h=\Delta \gamma$ remains finite. The radii $a$ and $b$ are nearly identical: $\varepsilon=b-a \ll a, b$. By writing $b=a+\varepsilon$, then substituting

$$
g(x) \approx g(a)+g^{\prime}(a)(x-a),
$$

and with subsequent integration and expansion to terms of the order $\varepsilon^{1 / 2}$ and $\varepsilon$, (3.157) can be represented in the following way:

$$
\begin{aligned}
h & \approx \frac{2}{\pi}[g(a)-d] \sqrt{\frac{2 \varepsilon}{a}}-\frac{4 \sigma_{0}}{\pi E^{*}} \varepsilon, \\
d & \approx g(a)-\frac{2 \sigma_{0} a}{E^{*}} \sqrt{\frac{2 \varepsilon}{a}}, \\
F_{N} & \approx 2 E^{*} \int_{0}^{a}[d-g(x)] \mathrm{d} x .
\end{aligned}
$$


From the first two equations, we obtain the indenter shape independent relationship:

$$
\frac{4 \sigma_{0} \varepsilon}{\pi E^{*}}=h
$$

Substituting (3.160) into (3.159) gives the previously established general equations of the JKR approximation for arbitrary rotationally symmetric profiles (compare to (3.27) and (3.28)):

$$
\begin{gathered}
d \approx g(a)-\sqrt{\frac{2 \pi a \Delta \gamma}{E^{*}}}, \\
F_{N} \approx 2 E^{*} \int_{0}^{a}[d-g(x)] \mathrm{d} x .
\end{gathered}
$$

\subsubsection{The DMT Limiting Case for an Arbitrary Rotationally Symmetric Body}

Setting $a=0$ in (3.157) yields:

$$
\begin{aligned}
h & =\frac{2}{\pi} \int_{0}^{b} \frac{g(x)}{\sqrt{b^{2}-x^{2}}} \mathrm{~d} x-d-\frac{4 \sigma_{0}}{\pi E^{*}} b, \\
0 & =E^{*} d+2 \sigma_{0} b, \\
F_{N} & =-\pi b^{2} \sigma_{0} .
\end{aligned}
$$

Applying the familiar MDR inverse transformation (see (2.7))

$$
f(b)=\frac{2}{\pi} \int_{0}^{b} \frac{g(x)}{\sqrt{b^{2}-x^{2}}} \mathrm{~d} x
$$

to the first two equations provides the relationship:

$$
f(b)+\frac{2 \sigma_{0} b}{E^{*}}\left(1-\frac{2}{\pi}\right)=h .
$$

The classic "DMT limiting case" stands for:

$$
f(b) \gg \frac{2 \sigma_{0} b}{E^{*}}\left(1-\frac{2}{\pi}\right) .
$$

It is assumed but not proven that the state $a=0$ corresponds to a loss of stability. In the following we will provide solutions for specific indenter shapes. 


\subsubsection{The Paraboloid}

In this case, the shape of the indenter is defined by the equation

$$
f(r)=\frac{r^{2}}{2 R}
$$

with the paraboloid radius $R$. The MDR transformed profile is:

$$
g(x)=\frac{x^{2}}{R}
$$

and (3.157) determining the radii $a$ and $b$, the normal force $F_{N}$, and the indentation depth $d$, with range $h$ and magnitude of the adhesive stress $\sigma_{0}$, take on the following form:

$$
\begin{aligned}
\frac{\pi}{2} h & =\left(\frac{b^{2}}{2 R}-d\right) \arccos \left(\frac{a}{b}\right)+\frac{a}{2 R} \sqrt{b^{2}-a^{2}}-\frac{2 \sigma_{0}}{E^{*}}(b-a), \\
d & =\frac{a^{2}}{R}-\frac{2 \sigma_{0}}{E^{*}} \sqrt{b^{2}-a^{2}} \\
F_{N} & =2 E^{*}\left(a d-\frac{a^{3}}{3 R}\right)-\sigma_{0}\left[-2 a \sqrt{b^{2}-a^{2}}+2 b^{2} \arccos \left(\frac{a}{b}\right)\right] .
\end{aligned}
$$

We introduce the following dimensionless variables:

$$
\tilde{a}:=\frac{a}{a_{c}^{\mathrm{JKR}}}, \quad \tilde{d}:=\frac{d}{\left|d_{c}^{\mathrm{JKR}}\right|}, \quad \tilde{F}_{N}:=\frac{F_{N}}{F_{A}^{\mathrm{JKR}}}, \quad \kappa:=\frac{b}{a}
$$

where the quantities $a_{c}^{\mathrm{JKR}}, d_{c}^{\mathrm{JKR}}, F_{A}^{\mathrm{JKR}}$ can be referenced in Sect. 3.5.3:

$$
\begin{gathered}
a_{c}^{\mathrm{JKR}}=\left(\frac{9 \pi R^{2} \Delta \gamma}{8 E^{*}}\right)^{1 / 3}, \quad d_{c}^{\mathrm{JKR}}=-\left(\frac{3 \pi^{2} R \Delta \gamma^{2}}{64 E^{* 2}}\right)^{1 / 3}, \\
F_{A}^{\mathrm{JKR}}=\frac{3}{2} \pi R \Delta \gamma .
\end{gathered}
$$

Equations (3.168) then take on the following form:

$$
\begin{aligned}
\frac{2}{3} \frac{1}{\Lambda}= & \tilde{a}^{2}\left[\frac{\kappa^{2}}{2} \arccos \left(\frac{1}{\kappa}\right)-\arccos \left(\frac{1}{\kappa}\right)+\frac{1}{2} \sqrt{\kappa^{2}-1}\right] \\
& +\frac{4}{3} \tilde{a} \Lambda\left[1-\kappa+\sqrt{\kappa^{2}-1} \arccos \left(\frac{1}{\kappa}\right)\right], \\
\tilde{d}= & 3 \tilde{a}^{2}-4 \tilde{a} \Lambda \sqrt{\kappa^{2}-1}, \\
\tilde{F}_{N}= & \tilde{a}^{3}-\tilde{a}^{2} \Lambda\left(\kappa^{2} \arccos \left(\frac{1}{\kappa}\right)+\sqrt{\kappa^{2}-1}\right),
\end{aligned}
$$


Fig. 3.29 Normalized normal force as a function of the normalized contact radius for the Maugis-adhesive contact of elastic spheres for different values of the Tabor parameter $\Lambda$. Thin solid lines denote the JKR and DMT limits

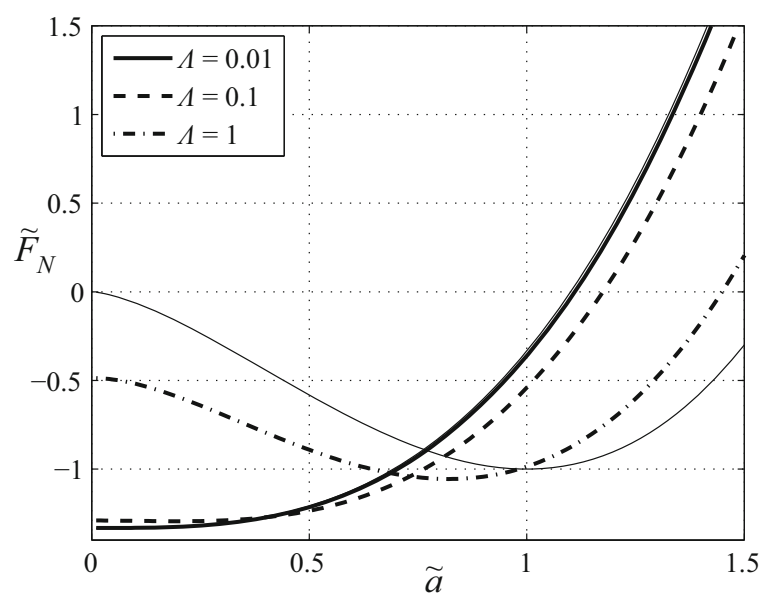

with

$$
\Lambda:=\left(\frac{3 R \sigma_{0}^{2}}{\pi h E^{* 2}}\right)^{1 / 3} .
$$

In Figs. 3.29 and 3.30 the dimensionless normal force as a function of the dimensionless contact radius or indentation depth (both relations are implicitly defined by (3.171)) is shown for different values of the Tabor parameter. It is apparent that the curves for small and large values of the Tabor parameter tend to be the respective DMT or JKR limit, denoted in the figures by thin solid lines. Interestingly the convergence towards the JKR limit seems to be more prone in the force-indentation depth-diagram, whereas the convergence towards the DMT limit appears to be "faster" (i.e., achieved for less extreme values of the Tabor parameter) in the force-contact radius-diagram. Also note that, whereas in the compressive branch of the force-indentation-curve there is no significant difference to the JKR limit already for $\Lambda=1$; the respective tensile branch still exhibits significant deviations from the JKR limit. Hence the adhesion range plays an important role for the stability (and, therefore, the hysteresis) of the contact even for large values of the Tabor parameter (for which most of the force-indentation-curve is practically the same as in the JKR limit), as was pointed out by Wu (2010) and Ciavarella et al. (2017).

For the limiting case of disappearing adhesion, (3.168) resolves to the Hertzian solution:

$$
\begin{aligned}
d & =\frac{a^{2}}{R}, \\
F_{N} & =2 E^{*}\left(a d-\frac{a^{3}}{3 R}\right)=\frac{4}{3} E^{*} \frac{a^{3}}{R} .
\end{aligned}
$$


Fig. 3.30 Normalized normal force as a function of the normalized indentation depth for the Maugis-adhesive contact of elastic spheres for different values of the Tabor parameter $\Lambda$. Thin solid lines denote the JKR and DMT limits

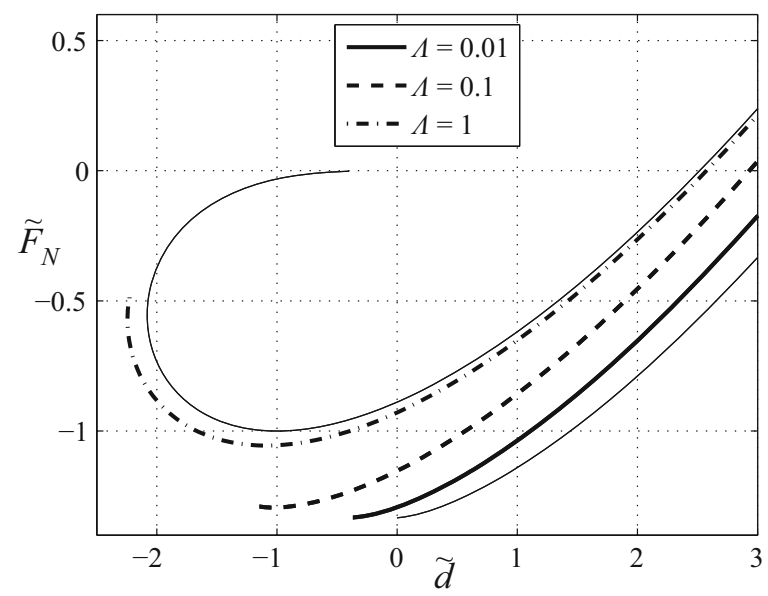

\section{The JKR Limiting Case}

With the aforementioned transition for $\sigma_{0} \rightarrow \infty$ and $h \rightarrow 0$, where $\sigma_{0} h=\Delta \gamma$, (3.168) yield:

$$
\begin{aligned}
h & \approx \frac{2}{\pi}\left(\frac{a^{2}}{R}-d\right) \sqrt{\frac{2 \varepsilon}{a}}-\frac{4 \sigma_{0} \varepsilon}{\pi E^{*}}, \\
d & \approx \frac{a^{2}}{R}-\frac{2 \sigma_{0} a}{E^{*}} \sqrt{\frac{2 \varepsilon}{a}}, \\
F_{N} & \approx \frac{4}{3} E^{*} \frac{a^{3}}{R}-4 \sigma_{0} a^{2} \sqrt{\frac{2 \varepsilon}{a}} .
\end{aligned}
$$

With (3.160), and taking into account $\Delta \gamma=\sigma_{0} h$, we obtain from (3.174) the JKR solution previously detailed in Sect. 3.5.3:

$$
\begin{aligned}
d & \approx \frac{a^{2}}{R}-\sqrt{\frac{2 \pi a \Delta \gamma}{E^{*}}}, \\
F_{N} & \approx \frac{4}{3} E^{*} \frac{a^{3}}{R}-\sqrt{8 \pi E^{*} a^{3} \Delta \gamma} .
\end{aligned}
$$

\section{The DMT Limiting Case}

For the case $a=0,(3.168)$ yield:

$$
\begin{aligned}
h & =\frac{b^{2}}{2 R}-d-\frac{4 \sigma_{0}}{\pi E^{*}} b, \\
d & =-\frac{2 \sigma_{0} b}{E^{*}}, \\
F_{N} & =-\pi b^{2} \sigma_{0} .
\end{aligned}
$$


The first two equations provide the relationship:

$$
\frac{b^{2}}{2 R}+\frac{2 \sigma_{0} b}{E^{*}}\left(1-\frac{2}{\pi}\right)-h=0,
$$

which can be solved for $b$ :

$$
b=-\frac{2 R \sigma_{0}}{E^{*}}\left(1-\frac{2}{\pi}\right)+\sqrt{\left[\frac{2 R \sigma_{0}}{E^{*}}\left(1-\frac{2}{\pi}\right)\right]^{2}+2 R h .}
$$

If the condition

$$
2 R h \gg \frac{4 R^{2} \sigma_{0}^{2}}{E^{* 2}}\left(1-\frac{2}{\pi}\right)^{2}
$$

is valid then $b \approx \sqrt{2 R h}$. Substituting into the third of the set of (3.176) then delivers the DMT result:

$$
F_{A, \mathrm{DMT}}=-F_{N}=2 \pi R h \sigma_{0}=2 \pi R \Delta \gamma
$$

It is easy to see that the condition (3.179)

$$
\frac{2 R \Delta \gamma^{2}}{E^{* 2} h^{3}}\left(1-\frac{2}{\pi}\right)^{2} \ll 1
$$

is identical to Tabor's criterion (3.3).

\section{Asymptotic Corrections for the JKR Solution}

We introduce the notation

$$
\varepsilon_{1}=\kappa-1
$$

and expand (3.171) to powers of $\varepsilon_{1}$ :

$$
\begin{aligned}
\frac{2}{3 \Lambda} & =\frac{4}{3} \tilde{a} \Lambda \varepsilon_{1}+\frac{4}{3} \sqrt{2} \tilde{a}^{2} \varepsilon_{1}{ }^{3 / 2}+\ldots, \\
\tilde{d} & =3 \tilde{a}^{2}-\tilde{a} \Lambda \sqrt{2}\left(4 \varepsilon_{1}{ }^{1 / 2}+\varepsilon_{1}{ }^{3 / 2}-\frac{1}{8} \varepsilon_{1}{ }^{5 / 2}+\ldots\right), \\
\tilde{F}_{N} & =\tilde{a}^{3}-\sqrt{2} \tilde{a}^{2} \Lambda\left(2 \varepsilon_{1}{ }^{1 / 2}+\frac{23}{6} \varepsilon_{1}^{3 / 2}+\frac{457}{240} \varepsilon_{1}^{5 / 2}+\ldots\right) .
\end{aligned}
$$

Utilizing second-order perturbation theory, solving the first equation for $\varepsilon_{1}$ yields:

$$
\varepsilon_{1}^{(1)} \approx\left(\frac{1}{2 \tilde{a} \Lambda^{2}}-\frac{1}{2 \tilde{a}^{1 / 2} \Lambda^{4}}+\ldots\right)
$$


Substituting into the second and third equations of (3.183) then gives:

$$
\begin{gathered}
\tilde{d}=3 \tilde{a}^{2}-4 \tilde{a}^{1 / 2}+\frac{1}{\Lambda^{2}}\left(2 \tilde{a}-\frac{5}{6} \tilde{a}^{-1 / 2}\right), \\
\tilde{F}_{N}=\tilde{a}^{3}-2 \tilde{a}^{3 / 2}-\frac{1}{\Lambda^{2}}\left(-\tilde{a}^{2}+\frac{23}{12} \tilde{a}^{1 / 2}\right) .
\end{gathered}
$$

Replacing 23 by 24 achieves a precision of approximately $5 \%$ for the perturbation term, providing the supremely simple approximation:

$$
\tilde{F}_{N} \approx\left(\tilde{a}^{3}-2 \tilde{a}^{3 / 2}\right)\left(1+\frac{1}{\tilde{a} \Lambda^{2}}\right), \quad \Lambda \gg 1 .
$$

In proximity to the critical radius $\tilde{a}=1$, we can write $\tilde{a}=1+\Delta \tilde{a}$ and expand to powers of $\Delta a$ :

$$
\begin{aligned}
\tilde{d} & \approx-1+\frac{7}{6 \Lambda^{2}}+\left(4+\frac{29}{12 \Lambda^{2}}\right) \Delta \tilde{a} \\
\tilde{F}_{N} & \approx-1-\frac{11}{12 \Lambda^{2}}+\frac{25}{24 \Lambda^{2}} \Delta \tilde{a}+\left(\frac{9}{4}+\frac{119}{96 \Lambda^{2}}\right) \Delta \tilde{a}^{2} .
\end{aligned}
$$

The force reaches a minimum for

$$
\frac{\mathrm{d} \tilde{F}_{N}}{\mathrm{~d}(\Delta \tilde{a})}=-\frac{9}{8 \Lambda^{2}}-\left(\frac{9}{2}+\frac{39}{16 \Lambda^{2}}\right) \Delta \tilde{a}=0 \Leftrightarrow \Delta \tilde{a}=-\frac{50}{216 \Lambda^{2}+119}
$$

Substituting this result into the second equation in (3.187), and subsequently expanding to $1 / \Lambda$ yields:

$$
\tilde{F}_{A}=-\tilde{F}_{N} \approx 1+\frac{11}{12 \Lambda^{2}},
$$

or (alternatively) in dimensional variables:

$$
\begin{aligned}
F_{A} & =\frac{3}{2} \pi R \sigma_{0} h+\frac{11}{24}\left(\frac{3 \pi^{5} E^{* 4} R h^{5}}{\sigma_{0}}\right)^{1 / 3} \\
& =\frac{3}{2} \pi R \Delta \gamma+\frac{11}{24}\left(\frac{3 \pi^{5} E^{* 4} R h^{6}}{\Delta \gamma}\right)^{1 / 3} .
\end{aligned}
$$

\subsubsection{The Profile in the Form of a Power-Law}

For an indenting, rotationally symmetric body with the general profile

$$
f(r)=c r^{n}, \quad n \in \mathbb{R}^{+},
$$


and positive numbers $n$ and a constant $c$, (3.157) take on the form:

$$
\begin{aligned}
h= & c b^{n}\left[1-\frac{2 \kappa(n)}{\pi(n+1)}\left(\frac{a}{b}\right)^{n+1}{ }_{2} \mathrm{~F}_{1}\left(\frac{1}{2}, \frac{n+1}{2} ; \frac{n+3}{2} ; \frac{a^{2}}{b^{2}}\right)\right] \\
& -\frac{2 d}{\pi} \arccos \left(\frac{a}{b}\right)-\frac{4 \sigma_{0}}{\pi E^{*}}(b-a), \\
0= & E^{*}\left[d-c \kappa(n) a^{n}\right]+2 \sigma_{0} \sqrt{b^{2}-a^{2}}, \\
F_{N}= & 2 E^{*}\left[d a-\frac{c \kappa(n)}{n+1} a^{n+1}\right] \\
& -\sigma_{0}\left[-2 a \sqrt{b^{2}-a^{2}}+2 b^{2} \arccos \left(\frac{a}{b}\right)\right] .
\end{aligned}
$$

Consistent with the notation of this subsection, we denote the radius of the contact area by $a$ and the zone radius of the adhesive interaction by $b>a$, the indentation depth by $d$, the normal force with $F_{N}$, the range by $h$, and the value of the adhesive stress by $\sigma_{0}$. We introduce:

$$
\kappa(n):=\sqrt{\pi} \frac{\Gamma(n / 2+1)}{\Gamma[(n+1) / 2]}
$$

and utilize the hypergeometric function:

$$
{ }_{2} \mathrm{~F}_{1}(a, b ; c ; z):=\sum_{n=0}^{\infty} \frac{\Gamma(a+n) \Gamma(b+n) \Gamma(c)}{\Gamma(a) \Gamma(b) \Gamma(c+n)} \frac{z^{n}}{n !} .
$$

Naturally, setting $n=2$ returns (3.168) of the preceding section.

The JKR limiting case of the above (3.192) was already discussed in detail in Sect. 3.5.7. As a final note, the adhesive force for this indenter shape in the DMT limiting case ( $h=f(b), a=0)$ is given by:

$$
F_{A}^{\mathrm{DMT}}=\frac{\pi \Delta \gamma}{c^{2 / n}} h^{\frac{2-n}{n}},
$$

with an effective surface energy of $\Delta \gamma=\sigma_{0} h$. It is apparent that the parabolic body with $n=2$ represents the sole case where this force is not explicitly dependent on the range of the adhesion.

\subsection{Adhesion According to Greenwood and Johnson}

We now consider the adhesive contact between a parabolic indenter $\tilde{z}=r^{2} /(2 R)$ and an elastic half-space. The surface displacement must correspond exactly to the indenter shape within the contact area, regardless of the type of adhesive interaction. Therefore, it is a quadratic function of radius $r$. Greenwood and Johnson 
(1998) rightly noted that this kinematic condition is met when the Hertzian pressure distribution is accompanied by an additional stress distribution. This additional distribution is, in turn, the difference of two Hertzian stress distributions with two varying indentation depths, and thus with the actual contact radius $a$ and another arbitrary fictional contact radius $c>a$, so that:

$$
\sigma_{z z}^{a d}(r)=\frac{2 E^{*}}{\pi R} \begin{cases}\sqrt{c^{2}-r^{2}}-\sqrt{a^{2}-r^{2}}, & r<a, \\ \sqrt{c^{2}-r^{2}}, & a<r<c, \\ 0, & r>c .\end{cases}
$$

A similar stress distribution was used far earlier already in the solution of the nonadhesive tangential contact of spheres to generate a constant displacement of a spherical domain (there will be more on this in the next chapter). Within the contact area, i.e., for $r \leq a$, this stress distribution (3.196) causes the constant displacement

$$
w(r)=\frac{1}{R}\left(a^{2}-c^{2}\right) .
$$

The additional stress (3.196) and the corresponding displacement (3.197) can be modified with an arbitrary factor $k$ for control of the indentation depth without changing the shape of the indenter. The entire stress distribution consisting of the original Hertzian distribution of radius $a$ and also the distribution (3.196) multiplied by the factor $k$ is given by:

$$
\sigma_{z z}(r)=-(1+k) \frac{2 E^{*}}{\pi R} \sqrt{a^{2}-r^{2}}+k \frac{2 E^{*}}{\pi R} \sqrt{c^{2}-r^{2}}, \quad r \leq a .
$$

Hertzian theory provides the distance $\Delta w$ between the two surfaces within the ring $a<r<c$ :

$$
\begin{gathered}
\Delta w(r)=(1+k) \frac{a^{2}}{\pi R}\left[\left(\frac{r^{2}}{a^{2}}-2\right) \arccos \left(\frac{a}{r}\right)+\frac{\sqrt{r^{2}-a^{2}}}{a}\right], \\
a<r<c .
\end{gathered}
$$

The stresses within the ring $a<r<c$

$$
\sigma_{z z}(r)=k \frac{2 E^{*}}{\pi R} \sqrt{c^{2}-r^{2}}, \quad a<r<c
$$

then describe the adhesive interactions between the surfaces. Combined with (3.199), they define the separation energy

$$
\begin{aligned}
\Delta \gamma & =\int \sigma \mathrm{d}(\Delta w)=\int_{c}^{a} \sigma_{z z}(r) \frac{\mathrm{d}(\Delta w)}{\mathrm{d} r} \mathrm{~d} r \\
& =k(k+1) \frac{2 E^{*}}{3 \pi R^{2}}(c-a)^{2}(c+2 a) .
\end{aligned}
$$

Minimizing the total energy provides equilibrium configuration. 
While the purely contact mechanical aspect of Greenwood's and Johnson's theory is far simpler than that of Maugis's theory, their model is based on certain assumptions with effects that are not immediately obvious. As an example, the implicitly introduced interaction not only depends on the distance between the surfaces but also on the entire configuration of the contact.

\section{References}

Borodich, F.M., Galanov, B.A.: Molecular adhesive contact for indenters of non-ideal shapes. In: ICTAM04, Abstracts book and CD-Rom Proceedings. IPPT PAN, Warsaw (2004)

Bradley, A.I.: The cohesive force between solid surfaces and the surface energy of solids. Philos. Mag. 13, 853-862 (1932)

Briggs, G.A.D., Briscoe, B.J.: The effect of surface topography on the adhesion of elastic solids. J. Phys. D Appl. Phys. 10(18), 2453-2466 (1977)

Ciavarella, M., Greenwood, J.A., Barber, J.R.: Effect of Tabor parameter on hysteresis losses during adhesive contact. J. Mech. Phys. Solids 98, 236-244 (2017)

Derjaguin, B.V., Muller, V.M., Toporov, Y.P.: Effect of contact deformations on the adhesion of particles. J. Colloid Interface Sci. 53(2), 314-326 (1975)

Dugdale, D.S.: Yielding of steel sheets containing slits. J. Mech. Phys. Solids 8(2), 100-104 (1960)

Gao, H., Yao, H.: Shape insensitive optimal adhesion of nanoscale fibrillar structures. Proc. Natl. Acad. Sci. U.S.A. 101(21), 7851-7856 (2004)

Greenwood, J.A., Johnson, K.L.: An alternative to the Maugis model of adhesion between elastic spheres. J. Phys. D Appl. Phys. 31(22), 3279-3290 (1998)

Griffith, A.A.: The Phenomena of Rupture and Flow in Solids. Philos. Trans. R. Soc. London Ser. A 221, 163-198 (1921)

Guduru, P.R.: Detachment of a rigid solid from an elastic wavy surface: theory. J. Mech. Phys. Solids 55(3), 445-472 (2007)

Hertz, H.: Über die Berührung fester elastischer Körper. J. Reine Angew. Math. 92, 156-171 (1882)

Heß, M.: Über die exakte Abbildung ausgewählter dreidimensionaler Kontakte auf Systeme mit niedrigerer räumlicher Dimension (2010). Dissertation, Technische Universität Berlin

Johnson, K.L., Kendall, K., Roberts, A.D.: Surface Energy and the Contact of Elastic Solids. Proc. R. Soc. London Ser. A 324, 301-313 (1971)

Kendall, K.: The adhesion and surface energy of elastic solids. J. Phys. D Appl. Phys. 4(8), 1186-1195 (1971)

Maugis, D.: Adhesion of spheres: the JKR-DMT-transition using a Dugdale model. J. Colloid Interface Sci. 150(1), 243-269 (1992)

Maugis, D., Barquins, M.: Adhesive contact of a conical punch on an elastic half-space. J. Phys. Lett. 42(5), 95-97 (1981)

Maugis, D., Barquins, M.: Adhesive contact of sectionally smooth-ended punches on elastic halfspaces: theory and experiment. J. Phys. D Appl. Phys. 16(10), 1843-1874 (1983)

Popov, V.L.: Surface profiles with zero and finite adhesion force and adhesion instabilities (2017). https://arXiv.org/abs/1707.07867. [cond-mat.soft]

Popov, V.L., Heß, M.: Method of dimensionality reduction in contact mechanics and friction. Springer, Heidelberg (2015). ISBN 978-3-642-53875-9

Spolenak, R., Gorb, S., Gao, H., Arzt, E.: Effects of contact shape on the scaling of biological attachments. Proc. R. Soc. London Ser. A 461, 305-319 (2005)

Tabor, D.: Surface forces and surface interactions. J. Colloid Interface Sci. 58(1), 2-13 (1977)

Thornton, C., Ning, Z.: A theoretical model for the stick/bounce behavior of adhesive, elasticplastic spheres. Powder Technol. 99(2), 154-162 (1998) 
Wu, J.J.: The jump-to-contact distance in atomic force microscopy measurement. J. Adhes. 86(11), 1071-1085 (2010)

Yao, H., Gao, H.: Optimal shapes for adhesive binding between two elastic bodies. J. Colloid Interface Sci. 298(2), 564-572 (2006)

Open Access This chapter is licensed under the terms of the Creative Commons Attribution 4.0 International License (http://creativecommons.org/licenses/by/4.0/), which permits use, sharing, adaptation, distribution and reproduction in any medium or format, as long as you give appropriate credit to the original author(s) and the source, provide a link to the Creative Commons license and indicate if changes were made.

The images or other third party material in this chapter are included in the chapter's Creative Commons license, unless indicated otherwise in a credit line to the material. If material is not included in the chapter's Creative Commons license and your intended use is not permitted by statutory regulation or exceeds the permitted use, you will need to obtain permission directly from the copyright holder.

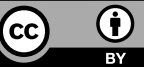

